\title{
DETEÇÃO DE CONDUTORES TABULARES SOB COBERTURA INDUTIVA
}

\author{
LÚCIA M.C. SILVA* \& MARCOS V.G. GALVÃO**
}

\begin{abstract}
DETECCION OF HALF-PLANE CONDUCTORS UNDER AN INDUCTIVE SUPERFICIAL LAYER. The effects of a superficial layer of relatively high induction number on the horizontal - loop electromagnetic (HLEM or Slingram) response to an isolated tilted half-plane are analysed by analog modeling. It was found that the superficial layer produces symmetry in the in-phase component, and therefore makes the tilted body seem vertical. Also, the superficial layer causes amplitude reduction and a clockwise phase rotation of the anomaly, which may result in the inversion of the quadrature component, base level shift of the profiles and appearance of an extra peak in the quadrature. The relations between these effects and the dip, induction number and depth of the half-plane as well as the required loop calibration are showed and compared with those for a superficial layer of weak-to-moderate induction number. The results show that it is possible to find information about the body in the noise, because the effects of the superficial layer are considered as noise. Such a paradox is discussed. Finally, the reported symmetry in the in-phase is analysed in respect to an analogous effect, that can occur for a body in galvanic contact with a conductive environment and it is associated with a current gathering phenomenon, also called negative screening.
\end{abstract}

Keywords: Overburden, HLEM, Slingram, analog model, scale-model, three-dimensional model, half-plane conductor, tabular conductor, electromagnetic method, EM.

\begin{abstract}
RESUMO Os efeitos de uma camada superficial de número de indução relativamente elevado sob a resposta de um condutor tabular inclinado e isolado, obtida com o sistema eletromagnético de bobinas horizontais coplanares (HLEM ou Slingram), são analisados por meio de modelamento analógico. Foi observado que a cobertura causa simetrização da componente em-fase, um efeito que conduz à interpretação de um condutor com mergulho superior ao real, bem como a redução da amplitude e da rotação no sentido horário da fase da anomalia, que pode gerar a reversão da componente em-quadratura, deslocamento da base do perfil e aparecimento de um pico extra na quadratura. As relações entre esses efeitos e o mergulho, número de indução e profundidade do corpo, bem como com o tipo de calibração das bobinas, são mostradas e comparadas com aquelas determinadas para camadas superficiais de número de indução fraco a moderado. Os resultados mostram que é possível encontrar informações sobre o corpo no ruído, já que os efeitos da camada superficial são considerados ruído. Tal paradoxo é discutido. Finalmente, a simetria da em-fase observada é analisada tendo em conta o efeito análogo que pode ocorrer quando corpo e ambiente condutivo encontram-se em contato galvânico, e é associado ao fenômeno de concentração de corrente, também conhecido como blindagem negativa.
\end{abstract}

Palavras-chaves: Manto de intemperismo, HLEM, Slingram, modelo analógico, modelo reduzido, modelo de três dimensões, condutor semiplano, condutor tabular, método eletromagnético, EM.

INTRODUÇÃO Em regiões tropicais e semi-áridas, é comum a presença de uma camada superficial que, devido a sua expressiva condutância e, portanto, acoplamento com os sistemas comuns de prospecção eletromagnética (EM), bem como com os possíveis condutores subsuperficiais, deve ser considerada na interpretação EM a fim de se evitar resultados errôneos (Paterson 1967, Parasnis 1971, Palacky 1975, Preston 1975, Lajoie \& West 1977, Palacky \& Kadekàru 1978 e 1979, Palacky \& Sena 1979).

Lowrie \& West (1965) e Silva \& Verma (1986) estudaram, por meio de modelamento analógico, os efeitos da camada superficial sobre as respostas de corpos tabulates do tipo semi-plano, obtidas com o sistema HLEM ou Slingram (arranjo coplanar horizontal), de uso recomendado quando esta camada ocorre (Palacky \& Sena 1979). Os dois primeiros autores verificaram que a camada de cobertura promove, na anomalia gerada por corpos verticais: deslocamento em relação à base $(0 \%)$, redução da amplitude e do giro da fase no sentido horário, podendo até gerar a reversão da quadratura. Silva \& Verma (1986) confirmaram a validade desses efeitos para semiplanos inclinados e mostraram o aparecimento de um pico extra na quadratura. Ademais, eliminara o deslocamento da anomalia em relação à base, calibrando o sinal de referência sobre a camada superficial, operação mais provável no campo.

A redução da amplitude e a rotação da fase da anomalia levam a interpretação EM, realizada com diagramas de Ar- gand que desprezam a cobertura, a apontar para um depósito mais profundo e de maior condutância do que o real. O pico extra dificulta enormemente a quantificação da anomalia, mas fornece guias importantes para a interpretação qualitativa seu aparecimento é associado ao afastamento do corpo da vertical e ao crescimento do número de indução do corpo e da camada superficial, além disso, quanto mais evidente for o pico extra, mais raso pode estar o corpo.

Os dois trabalhos descrevem efeitos de coberturas com número de indução ou parâmetro de resposta $(\alpha)$ máximo igual a 1,0 , sendo $\alpha-\alpha \sigma 2 \pi \mathrm{ft} \ell$, onde $\mu$ - permeabilidade magnética $\left(=4 \pi \times 10^{-7} \mathrm{H} / \mathrm{m}\right.$, para modelos isentos de materiais ferromagnéticos), $\sigma=$ condutividade elétrica $(\mathrm{S} / \mathrm{m}), \mathrm{f}=$ freqüência $(\mathrm{Hz}), \mathrm{t}=$ espessura do corpo $(\mathrm{m}) \mathrm{e} \ell=$ distância entre as bobinas (m). Algumas características do problema, contudo, mostram que a camada superficial pode atingir, não raramente, número de indução relativamente elevado. Na Austrália, por exemplo, a condutância ( $\sigma$ ) da cobertura atinge $10 \mathrm{~S}$ (Preston 1975); nas regiões brasileiras, com exceção da Amazônia, esse valor cai para cerca de 1 S (Palacky \& Kadekaru 1978); para a região não estudada, no entanto, o manto pode desenvolver-se consideravelmente e atingir espessuras superiores a 200 m (Issler \& Silva 1980), mas a lixiviação intensa reduz a condutividadè dessa camada. Por outro lado, a resposta à cobertura cresce muito mais rapidamente com uma diminuição moderada do espaçamento entre as bobinas do que com

* Laboratório de Modelamento Analógico EM, Departamento de Geofísica, Centro de Geociências, Universidade Federal do Pará, Caixa Postal 1611, CEP 66059, Belém, PA, Brasil, e Institui fuer Meteoroiogie und Geophysik, Johann Wolfgang Goethe Universitaet. Feldbergstrasse 47, D-6000 Frankfurt a.M.-1, Alemanha

** SERSUL/DIRGEO/DESUD/PETROBRÁS, Av. Hias Agostinho, 665, Ponta de Impetiba, Macaé, RJ, Brasil 
um grande aumento da freqüência; conseqüentemente, o efeito de blindagem por ela provocado é minimizado preferencialmente com grandes espaçamentos entre as bobinas ao invés de freqüências excessivamente baixas (Parasnis 1971).

O objetivo primeiro deste trabalho foi o levantamento, por meio de modelamento analógico, de respostas EM afetadas por cobertura de número de indução elevado $(>1,0)$, bem como a análise sistemática dessas respostas com vistas à definição de guias para o trabalho de interpretação.

ARRANJO EXPERIMENTAL O corpo mineralizado e a camada superficial foram simulados por folhas metálicas não-magnéticas dispostas a uma profundidade normalizada com distância entre as bobinas $(\mathrm{h} / \ell)$ de, respectivamente, $0,2-$ 0,3-0,5 e 0,1, conforme é indicado na figura 1 (a e b) e na tabela 1. Na figura 1c, em adicional, é mostrada uma seção geológica possível para o problema. Para uma escala de redução de $5.000: 1$, por exemplo, a chapa de alumínio de 0,29 $\mathrm{mm}$ pode representar um corpo mineralizado com cobre (sulfetos) de 24,5 $\mathrm{S}$ de condutância $(\mathrm{t}=12,08 \mathrm{~m}$ e $\sigma=2,03 \mathrm{~S} / \mathrm{m})$, capeado por manto de intemperismo de $33,83 \mathrm{~m}$ de espessura e resistividade $(=1 / \sigma)$ igual a $11,86 \mathrm{ohm} . \mathrm{m}$, mas sem contato galvânico com este (isto é, profundidade do corpo > 33,83 $\mathrm{m}$ ), sendo a freqüência de operação do sistema e a distância entre as suas bobinas de $444 \mathrm{~Hz}$ e $150 \mathrm{~m}$, respectivamente. O mergulho do corpo $(\theta)$ no experimento assume os seguintes valores: $90^{\circ}, 75^{\circ}, 60^{\circ}, 45^{\circ}$ e $30^{\circ}$.

Os perfis foram realizados sobre o conjunto corpo + manto, com a calibração do sinal de referência com as bobinas sobre a cobertura condutiva (CAL.SOBRE), e na ausência da mesma (CAL.FORA), bem como na presença unicamente do corpo, com uma precisão de cerca de $95 \%$. A comparação de
Tabela 1 - Características e combinações das folhas metálicas usadas na simulação.

Table I - Characteristics and combinations of the metalic foils used in the simulation.

\begin{tabular}{c|c|c|c}
\hline MATERIAL & $\sigma\left(10^{6} \mathrm{~S} / \mathrm{m}\right)$ & $1\left(10^{3} \mathrm{~m}\right)$ & $\alpha_{f=673 H_{1}}$ \\
\hline AÇO $^{*}$ & 1,367 & 0,812 & 1,50 \\
AÇO & 1,418 & 2,570 & 4,59 \\
AI & 33,367 & 0,290 & 12,89 \\
AI & 30,470 & 0,514 & 20,67 \\
AI & 33,126 & 1,008 & 44,50 \\
& & & \\
\hline
\end{tabular}

* Utilizado para simulação do manto de intemperismo

alguns resultados com os de Lowrie \& West (1965) confirmam essa estimativa.

A teoria de modelamento analógico EM e os sistemas eletrônico e mecânico utilizados no presente trabalho são apresentados, com detalhes, em Silva (1981).

RESULTADOS Os 180 perfis produzidos permitiram reconhecer um novo efeito da cobertura - a simetrização da anomalia em fase obtida sobre corpos inclinados - isto é, sua aproximação da resposta de um condutor vertical.

(o)
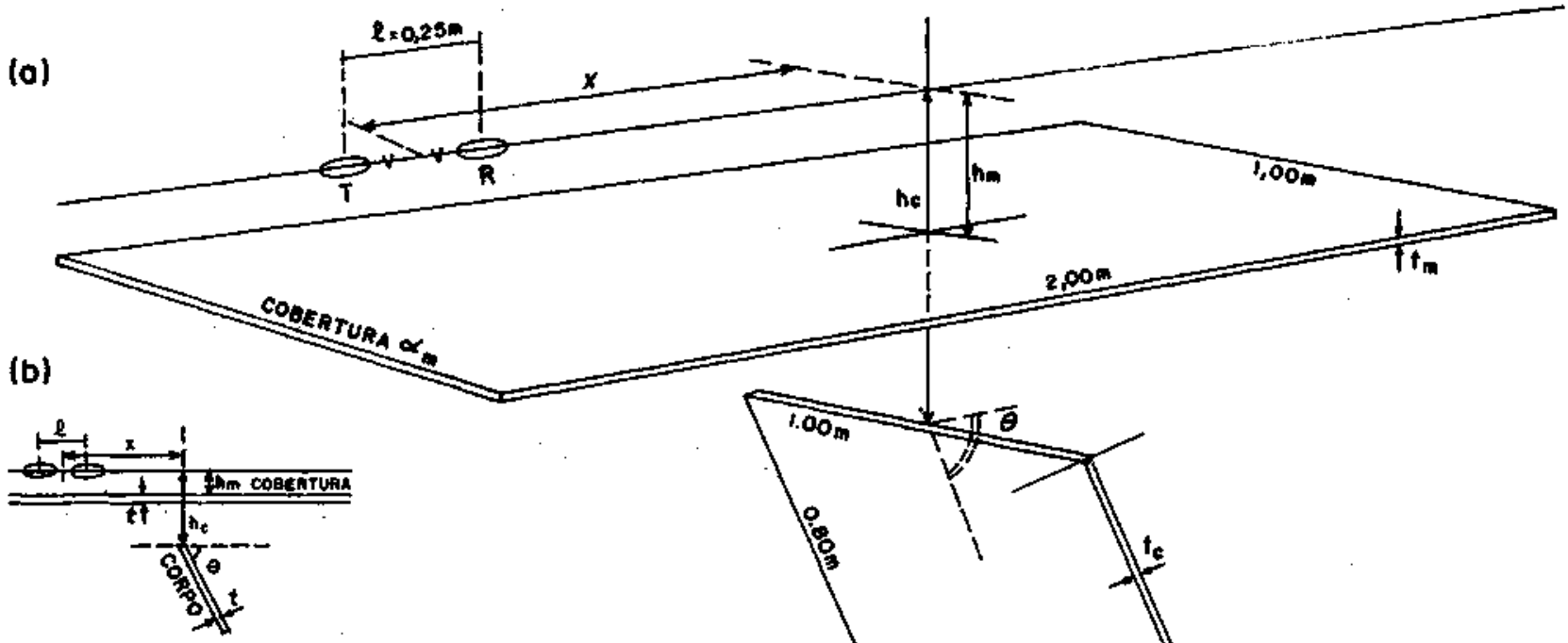

(c)

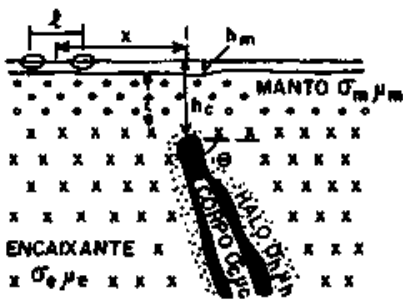

$\sigma_{\mathrm{m}}<\ll<\sigma_{c}$

$\sigma_{h}, \sigma_{h}$ desprezivets

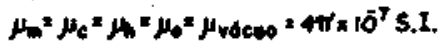

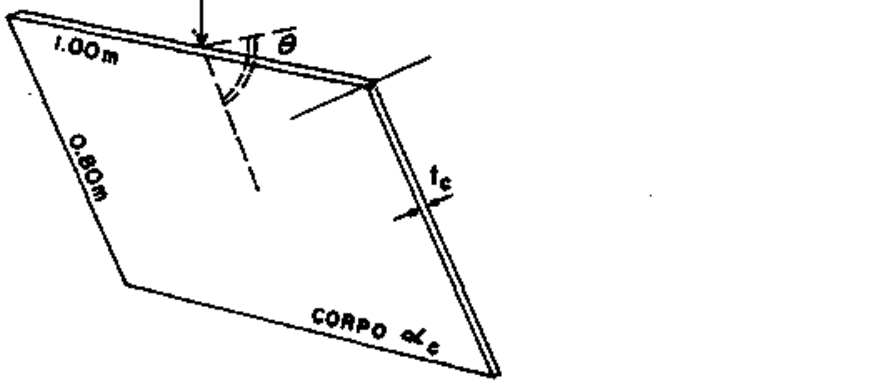

LEGENOA

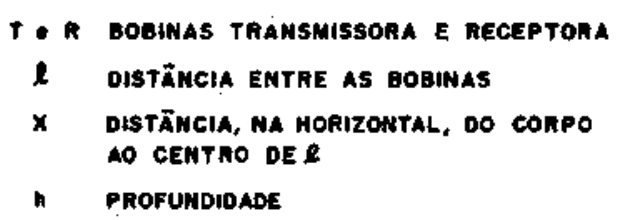

- MEROULhO

$\alpha$ MÚMERo de IMOUÇ̃̃o

o conputividade elétrica

a PERhea bilioade hagnética

t Espessuna

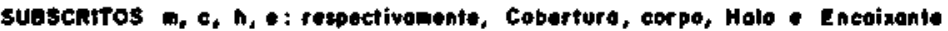

Figura 1 - Croqui do experimento: a. perspectiva; b. seção; c. exemplo de seção geológica simulada Figure 1 - Schematic view of the scale model: a. perspective; b. section; c. example of a geologic model simulated 
Arelação da simetrização e demais efeitos da cobertura com a variação dos parâmetros do alvo e do tipo de calibração é apresentada a seguir. Os elementos de anomalia utilizados são descritos na figura 2. A quantificação adotada para respostas com pico extra (Silva 1981) é explicada na figura 3. Essa quantificação permite a construção de diagramas de Argand ou, simplesmente, AIDs ("anomaly index diagrams") considerando-se os valores pico-a-pico tomados tanto do lado a favor do mergulho (PPF), quanto do lado contrario (PPQ

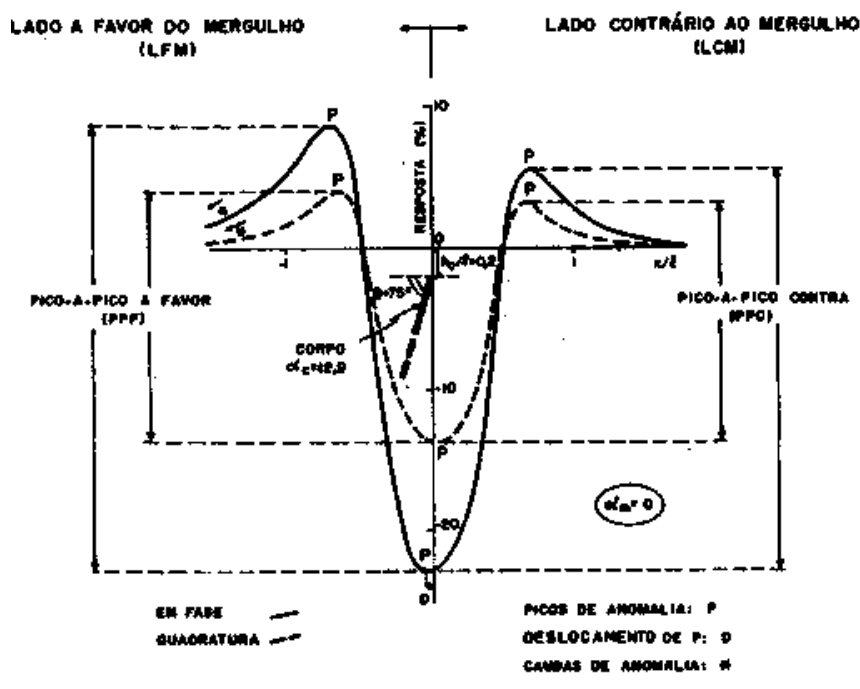

Figura 2 - Elementos da anomalia HLEM adotados Figure 2 - HLEM anomaly elements used in this work. Note: PPF - peakto-peak of the down dip side; $P P C=$ peak-to-peak of the up dip side

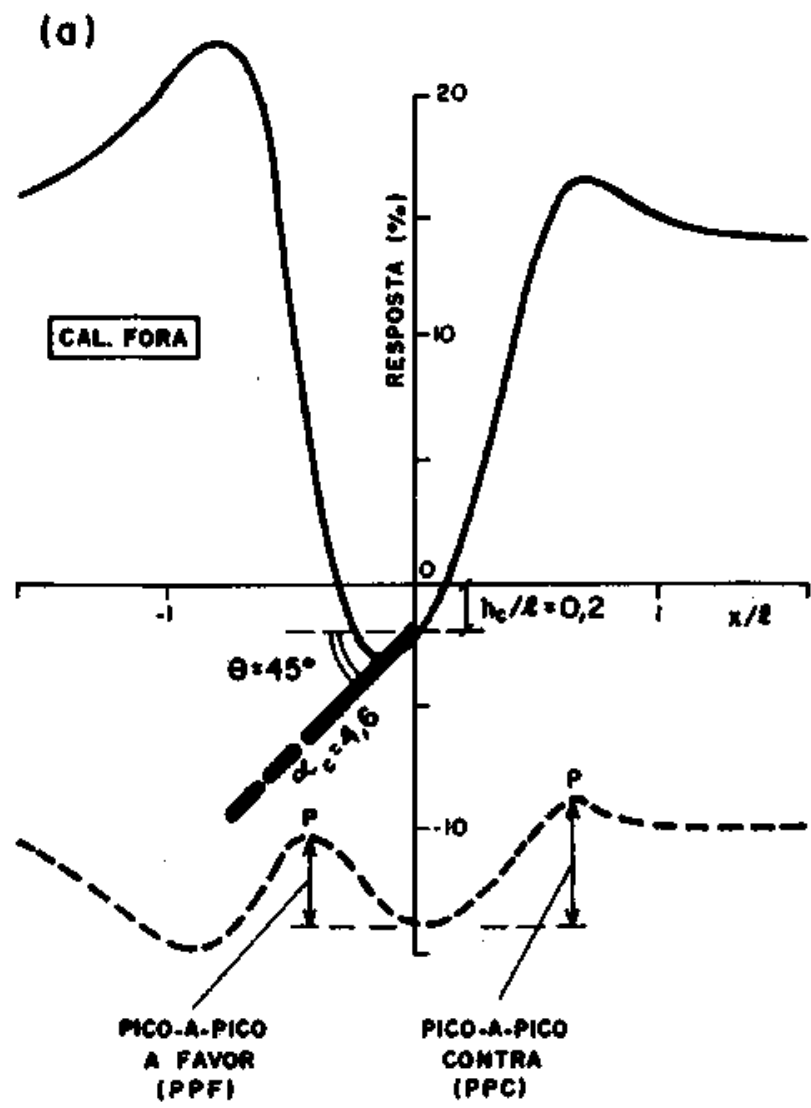

Efeitos da cobertura $\mathbf{x}$ mergulho do corpo $(\theta) \quad \grave{A}$ medida que o corpo se afasta da vertical e, portanto, da posição de mínimo acoplamento com o sistema de bobinas horizontais coplanares, a amplitude da resposta cresce segundo taxas diferentes para ambos os seus lados, de acordo com o afastamento bobinas-corpo, e a anomalia torna-se assimétrica; a ampliação da componente em-fase, especialmente no lado a favor do mergulho, é a principal responsável pela assimetria que se forma (Figs. 4a e $4 b$ ).

Existindo o manto, a assimetria da real provocada por $\theta$ pouco se desenvolve (Fig. $4 \mathrm{a}_{1}$ ). A quadratura pode ser substancialmente modificada, seja pela sua reversão e/ou aparecimento do pico extra, este ultimo efeito favorecido pela diminuição de $\theta$ (Fig. $4 \mathrm{~b}_{1}$ ). A figura $\mathrm{S}$ mostra que o manto torna os valores pico-a-pico da componente em-fase menos sensíveis a variações de 6 , enquanto com os valores da quadratura ocorre o contrario. A avaliação do mergulho do condutor a partir dos procedimentos convencionais, que têm por base a assimetria do perfil provocada por $\theta$, como o de Strangway (1966), conduzem a resultados.errôneos. Algumas feições do perfil podem, contudo, auxiliar na avaliação do mergulho como, por exemplo, sem ou com a cobertura: 1. as componentes atingem a base do perfil ( $0 \%$ com CAL.SOBRE) mais suavemente no sentido do mergulho, formando a chamada "cauda" da anomalia (Fig. 4); 2. a área sob a parte positiva da anomalia em-fase é maior do lado em que o condutor mergulha (Fig. 4a e $4 a_{1}$ ); 3. o deslocamento do pico negativo da anomalia em-fase aponta no sentido do mergulho do corpo (Figs. $4 \mathrm{a}$ e $4 \mathrm{a}_{1}$ ) - com a quadratura ocorre o mesmo, se inexiste a cobertura e o condutor é fraco ou moderado (Nair et al 1968) e se a camada superficial está presente, mas há inversão da componente e o pico extra apresenta-se pouco desenvolvido (Silva 1981) (Fig. 4b b $_{1}$.

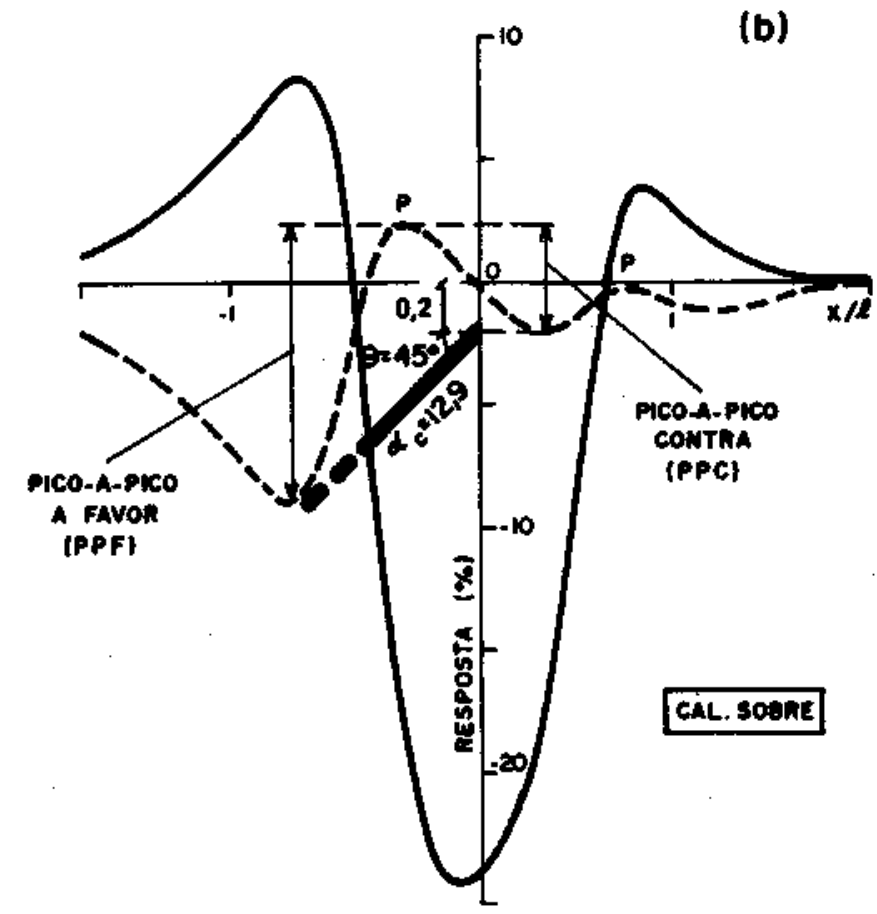

Figura 3 - Quantificação de respostas com pico extra. Perfil com quadratura: a, sem inversão (P "positivo" $L F M<P$ "positivo" $L C M)$; b. com inversão ( $P$ "positivo" $L F M>P$ "positivo" $L C M$ )

Figura 3 - Quantification of anomalies with an extra peak in the quadrature component. Profile with quadrature: a. not inverse ("positive" peak P of the down dip side $<$ "positive" peak P of the up dip side); b. inverse (vice versa). Note: CAL. FORA = calibration of the loops realized without overburden; CAL. SOBRE $=$ calibration of the loops with overburden 

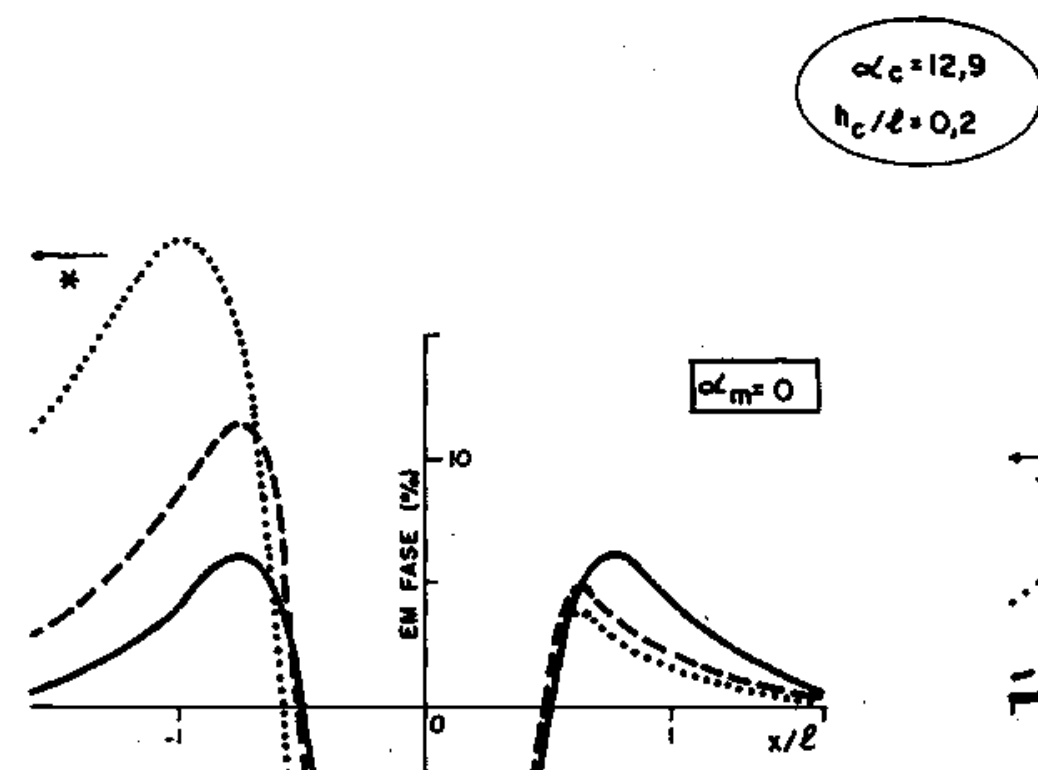

(0)
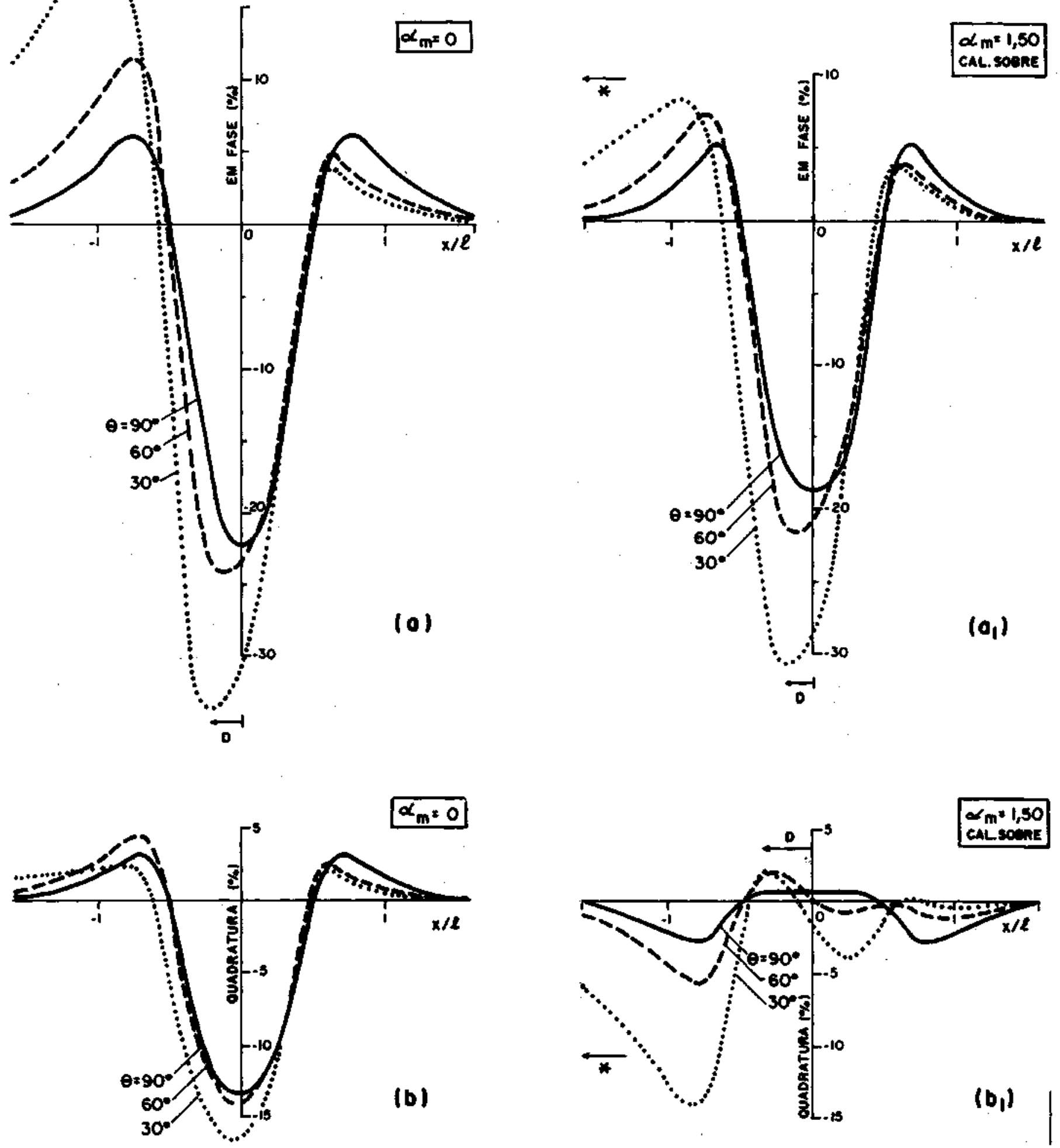

Figura 4 - Variação das respostas com o megulho ( $\theta$ ) do corpo: $\boldsymbol{a}$ e $\mathrm{a}_{1}$, em-fase; $\boldsymbol{b}$ e $\boldsymbol{b}_{1}$. em-quadratura. Subscrito 1 denota a presença da cobertura $\left(\alpha_{\mathrm{m}}=1,5\right)$

Figure 4 - Variation of response profile with dip angle of the body $(\theta)$. a and $\mathbf{a}_{\mathbf{1}}$. in-phase; $\mathbf{b}$ and $\mathbf{b}_{\mathbf{1}}$. quadrature. Subscript 1 means that overburden is present $(\alpha \mathrm{m}=1.5)$ 

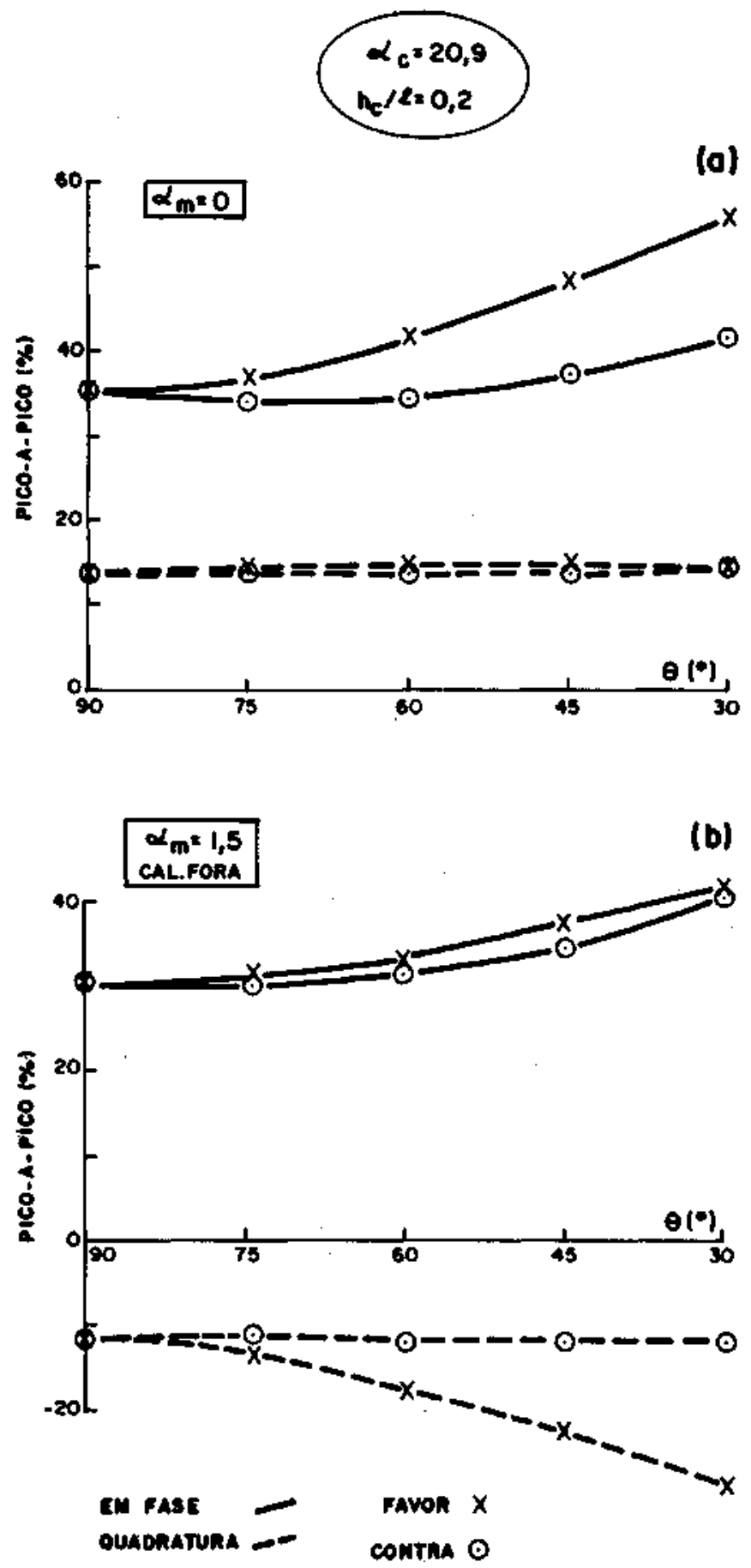

Figura 5 - Variação dos valores pico-a-pico corri o mergulho do corpo $(\theta)$. a. sem a cobertura $(\alpha \mathrm{m}=0)$ e b. com a cobertura $(\alpha \mathrm{m}=1,5)$

Figure 5 - Variation of peak-to-peak values of dip angle of the body $(\theta)$. a. when there is no overburden $\left(\alpha_{m}=0\right)$ and $b$. when the overburden has a response parameter $\alpha_{\mathrm{m}}=1.5$

Nos AIDs, variações na amplitude e na fase da anomalia, bem como suas conseqüências práticas, são facilmente identificadas. O manto de intemperismo causa a redução da amplitude e a rotação no sentido horário da fase da anomalia (Figs. 6 a 9); a interpretação realizada com diagramas que desprezam esta cobertura apontará, então, para um depósito mais profundo e de maior condutância do que o real, seja o corpo vertical (Lowrie \& West 1965) ou inclinado (Silva
\& Verma 1986). À medida que o corpo se afasta da vertical, contudo, a redução da amplitude e o giro na fase da anomalia provocados pela cobertura diminuem, quando se considera os valores PPC (em média, respectivamente, cerca de $-5 \%$ e $-7^{\circ}$, entre $\theta-60^{\circ}$ e $30^{\circ}$ com $\alpha_{m}=1,5$, independente da calibração (Figs. 7 e 9). As variações em amplitude e fase devidas à cobertura são sempre maiores para os valores PPF (Figs. 6a 9) e variam, com $\theta$, de uma fração muito pequena (em média, respectivamente, cerca de $-0,5 \%$ e $+1,5^{\circ}$ entre $\theta-60^{\circ}$ e $30^{\circ}$ com $\alpha_{m}-1,5$, independente da calibração) (Figs. 6 e 8). Conseqüentemente, a interpretação com um AID para parâmetro de resposta do manto inferior ao real apontará para um depósito mais profundo e de maior condutância do que o real, mas os erros serão menores quando forem utilizados diagramas PPC e mais o condutor se afastar da vertical.

Como cada diagrama é construído com as respostas de condutores de igual mergulho, é útil analisar o comportamento dos diagramas com $\theta$. Recombinando-se os diagramas das figuras 6 a 9 é possível observar que, inexistindo o manto, a amplitude da anomalia cresce e sua fase experimenta um giro no sentido horário, à medida que o condutor afasta-se da vertical (Figs. 10 e 11); com a camada superficial ocorre o mesmo, exceto com os valores PPC que giram no sentido anti-horário (Figs. 12 e 13). A interpretação com um diagrama para condutor com mergulho superior ao real apontará, conseqüentemente, para um depósito mais raso e de maior condutância do que o verdadeiro, exceto para AIDs-PPC construídos com o manto presente, quando a condutância encontrada será inferior à real. Como os valores PPF são os mais sensíveis à variação de 6 (Fig. 5), é preferível trabalhar com os AIDs-PPC quando o mergulho do alvo é duvidoso. Os diagramas PPC para $90^{\circ}<\theta<60^{\circ}$ podem ser utilizados indistintamente sem conduzir a interpretação dos parâmetros a erro considerável.

Efeitos da cobertura $x$ número de indução do corpo $\left(\alpha_{c}\right) \mathrm{O}$ aumento do parâmetro Oc favorece a simetrização da em-fase e tende a eliminar o pico extra em benefício da reversão da quadrature (Fig. 14). Silva \& Verma (1986), contudo, mostram que na faixa $\mathrm{O}<\alpha_{\mathrm{cm}}<1,0$, a probabilidade de aparecimento do pico extra cresce com $\alpha_{c}$.

As respostas pico-a-pico, para diferentes números de indução do corpo mostradas na figura 15, fazem ganhar sentido os resultados anteriormente apresentados. Sem o manto, as respostas são semelhantes às do circuito de três espiras de Grant \& West (1965): para a faixa de ot investigada, a proporção que este parâmetro cresce, as respostas em quadratura tendem a zero enquanto as respostas em-fase aumentam, atingindo diferentes limites indutivos, de acordo com o lado da anomalia - é, portanto, na componente em-fase que a assimetria introduzida pela variação de $\theta$ se desenvolve (Fig. 15a). O modelo corpo e manto tem um comportamento diferente: as respostas em-fase atingem os limites indutivos com um menor $\alpha_{c}$ e estes limites se desenvolvem a uma amplitude reduzida e muito próxima (podendo coincidir, como verificado); as respostas em-quadratura atingem valores negativos diferentes de acordo com o lado da anomalia - é, pois, na quadrature que os efeitos ligados a $\theta$ se manifestam (Fig. 15b)

Os AIDS mostram que ao aumento de $\alpha_{c}$ corresponde um aumento, tanto na amplitude como na fase (giro no sentido horário) da anomalia, de uma taxa aproximadamente constante (Figs. 6 a 13). Em presença da cobertura, contudo, com a quantificação adotada, as curvas de isovalores de número de indução podem tornar-se bastante próximas, implicando na perda de resolução do número de indução, bem como sofrem uma descontinuidade próximo da troca de sinal da quadrature (Figs. 6 a 9); ambos os casos refletem a interação dos efeitos de pico extra e reversão da quadrature. 


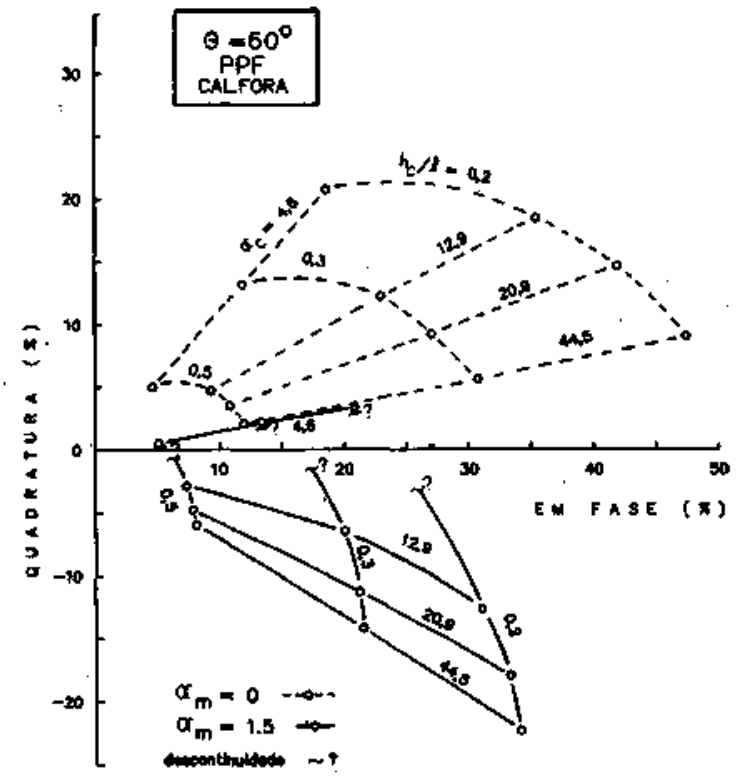

Figura 6 - Diagramas de valores pico-a-pico do lado a favor do mergulho do corpo para $\theta=60^{\circ}$ e $\alpha_{\mathrm{m}},=O$ e 1,5 (calibração fora do manto)

Figure 6 - Argand diagrams (peak-to-peak excursion of the components in the down dip side - PPF) for a body with dip angle $\theta-60^{\circ}$ when there is no overburden $\left(\alpha_{\mathrm{m}}=0\right)$ and when the overburden has a response parameter $\alpha_{m}=1.5$ (calibration of the loops realized without the overburden - CAL. FORA)

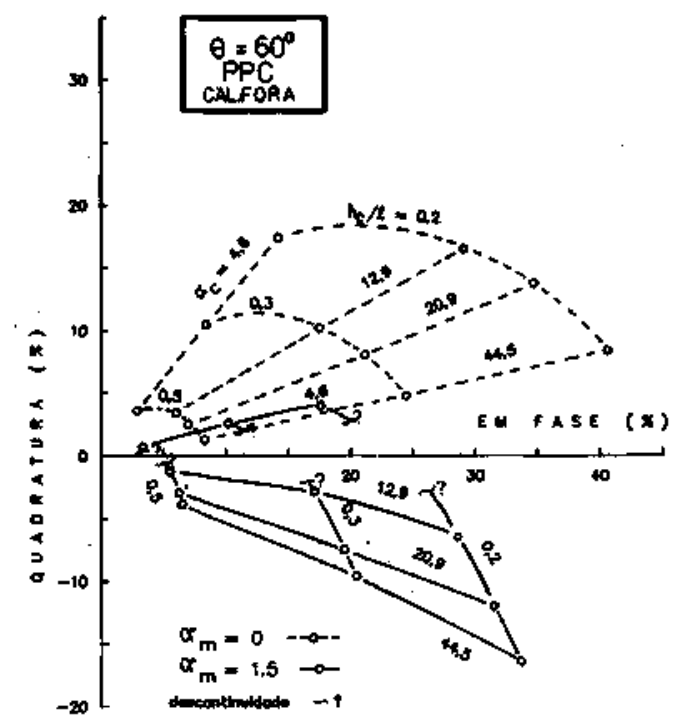

Figura 7 - Diagramas de valores pico-a-pico do lado contrário ao mergulho do corpo para $\theta=60^{\circ}$ e $\alpha_{\mathrm{m}}=0$ e 1,5 (calibração fora do manto)

Figure 7 - Argand diagrams (peak-to-peak excursion of the components in the up dip side - PPQ for a body with dip angle $\theta=60^{\circ}$ when there is no overburden $\left(\alpha_{\mathrm{m}}=0\right)$ and when the overburden has a response parameter $\alpha_{\mathrm{m}}$ $=1.5$ (calibration of the loops realized without the overburden - CAL. FORA)

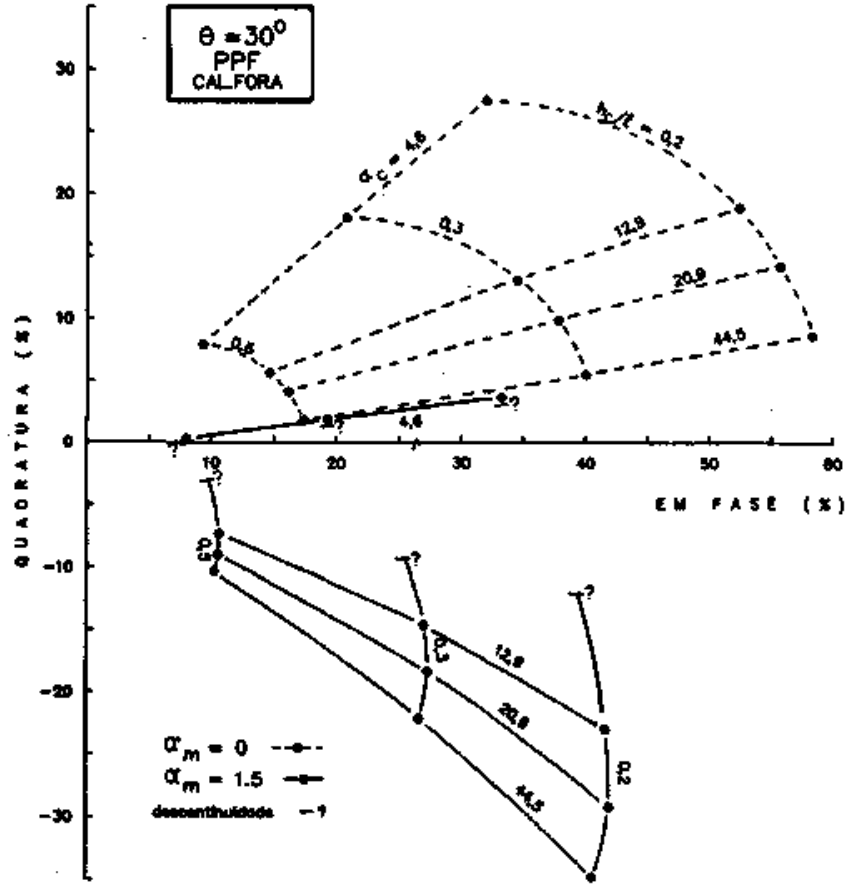

Figura 8 - Diagramas de valores pico-a-pico do lado a favor do mergulho do corpo para $\theta=30^{\circ}$ e $\alpha_{\mathrm{m}}=0$ e 1,5 (calibração fora do manto)

Figure 8 - Argand diagrams (peak-to-peak excursion of the components in the down dip side - PPF) for a body with dip angle $\theta-30^{\circ}$ when there is no overburden $\left(\alpha_{\mathrm{m}}=0\right)$ and when the overburden has a response parameter $\alpha_{m}=1.5$ (calibration of the loops realized without the overburden - CAL. FORA)

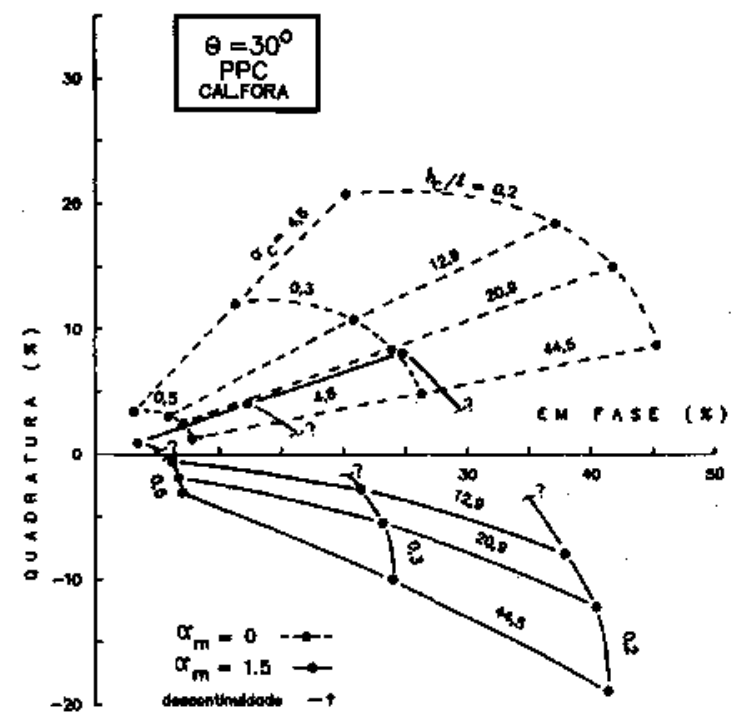

Figura 9 - Diagramas de valores pico-a-pico do lado contrário ao mergulho do corpo para $\theta=30^{\circ}$ e $\alpha_{\mathrm{m}}=0$ e 1,5 (calibração fora do manto)

Figure 9 - Argand diagrams (peak-to-peak excursion of the components in the up dip side - PPC) for a body with dip angle $\theta=30^{\circ}$ when there is no overburden $\left(\alpha_{m}=0\right)$ and when the overburden has a response parameter $\alpha_{m}$ $=1.5$ (calibration of the loops realized without the overburden - CAL. FORA) 


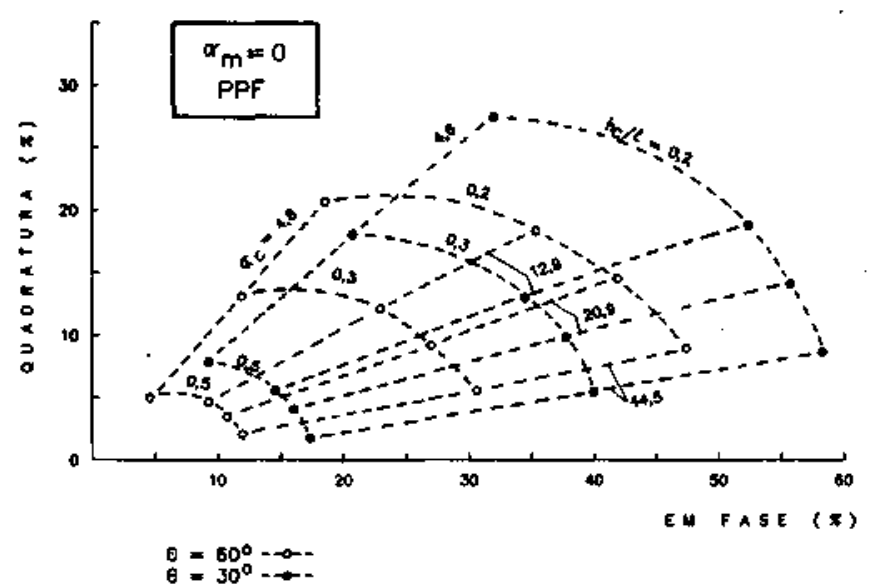

Figura 10 - Diagramas de valores pico-a-pico do lado a favor do mergulho do corpo para $\alpha_{\mathrm{m}}=0$ e $\theta=60^{\circ}$ e $30^{\circ}$ (calibração fora do manto)

Figure 10 - Argand diagrams (peak-to-peak excursion of the components in the down dip side - PPF) for a body with dip angle $\theta=60^{\circ}$ and $30^{\circ}$ when there is no overburden $\left(\alpha_{\mathrm{m}}=0\right)$ (calibration of the loops without the overburden - CAL. FORA)

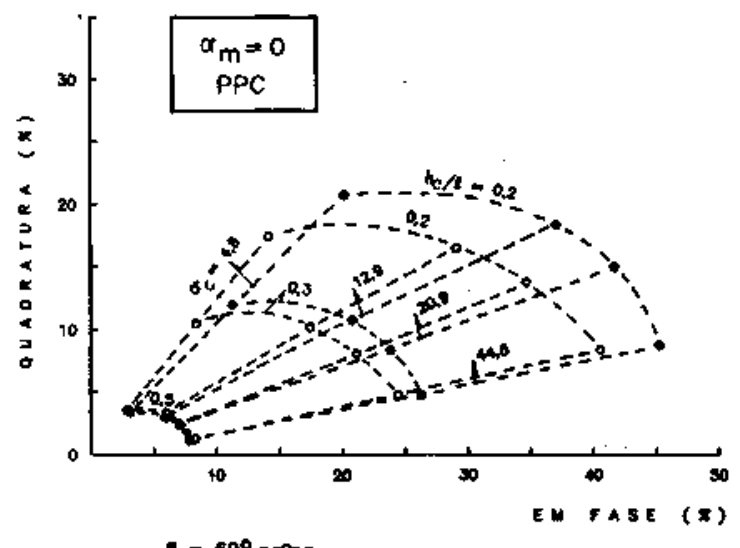

$\theta=60^{\circ}-\infty-$

Figura 11 - Diagramas de valores pico-a-pico do lado contrário ao mergulho do corpo para $\alpha_{m}=0$ e $\theta=60^{\circ}$ e $30^{\circ}$ (calibração fora do manto)

Figure 11 - Argand diagrams (peak-to-peak excursion of the components in the up dip side - PPC) for a body with dip angle $\theta=60^{\circ}$ and $30^{\circ}$ when there is no overburden $\left(\alpha_{\mathrm{m}}=0\right)$ (calibration of the loops without the overburden CAL. FORA)

Efeitos da cobertura $x$ profundidade do corpo (hc/C) A indutância mútua entre dois circuitos, dada pela fórmula de Neumann, é inversamente proporcional à distância entre os mesmos. Quanto mais profundo o corpo, portanto, menor sua interação com o sistema de bobinas. Conseqüentemente, a profundidade é inversamente proporcional à simetrízação da em-fase e à formação do pico extra na quadratura, produtos de uma interação maior entre o conjunto corpo, manto e bobinas introduzidas pela inclinação do corpo. Em outras

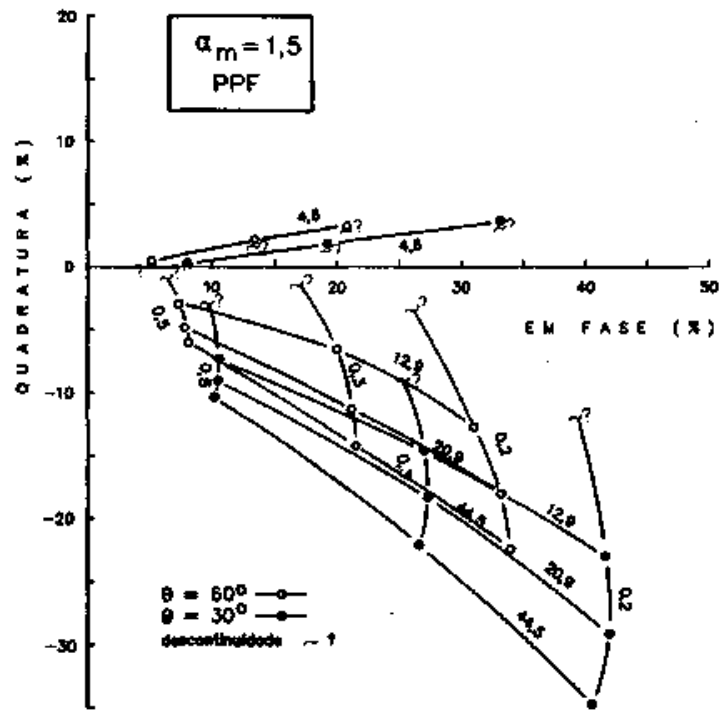

Figura 12 - Diagramas de valores pico-a-pico do lado a favor do mergulho do corpo para $\alpha_{\mathrm{m}}=1,5$ e $\theta=60^{\circ}$ e $30^{\circ}$ (calibração fora do manto)

Figure 12 - Argand diagrams (peak-to-peak excursion of the components in the down dip side - PPF) for a body with dip angle $\theta=60^{\circ}$ and $30^{\circ}$ when the overburden has a response parameter $\alpha_{m}=1.5$ (calibration of the loops without the overburden - CAL. FORA)

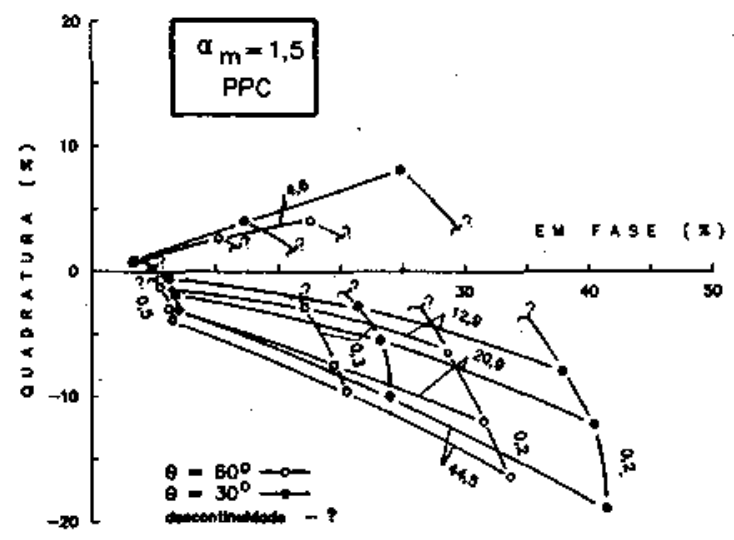

Figura 13 - Diagramas de valores pico-a-pico do lado contrário ao mergulho do corpo para $\alpha_{\mathrm{m}}=1,5$ e $\theta=60^{\circ}$ e $3 O^{\circ}$ (calibração fora do manto)

Figure 13 - Argand diagrams (peak-to-peak excursion of the components $b$ the up dip side - PPC) for a body with dip angle $\theta=60^{\circ}$ and $30^{\circ}$ when the overburden has a response parameter $\alpha_{\mathrm{m}}=1.5$ (calibration of the loops without die overburden - CAL. FORA)

palavras, o aumento da profundidade minimiza os efeitos de simetrízação da em-fase e da formação do pico extra na quadratura, podendo o pico extra ser substituído integralmente pela reversão da quadratura (Fig. 16).

Nos AIDS, o aumento da profundidade do condutor desloca o valor pico-a-pico para posições de menor amplitude segundo curvas de mesma fase, salvo quando existe o manto, quando variações de fase, com a quantificação adotada, são observadas especialmente nas respostas dos condutores mais rasos, 


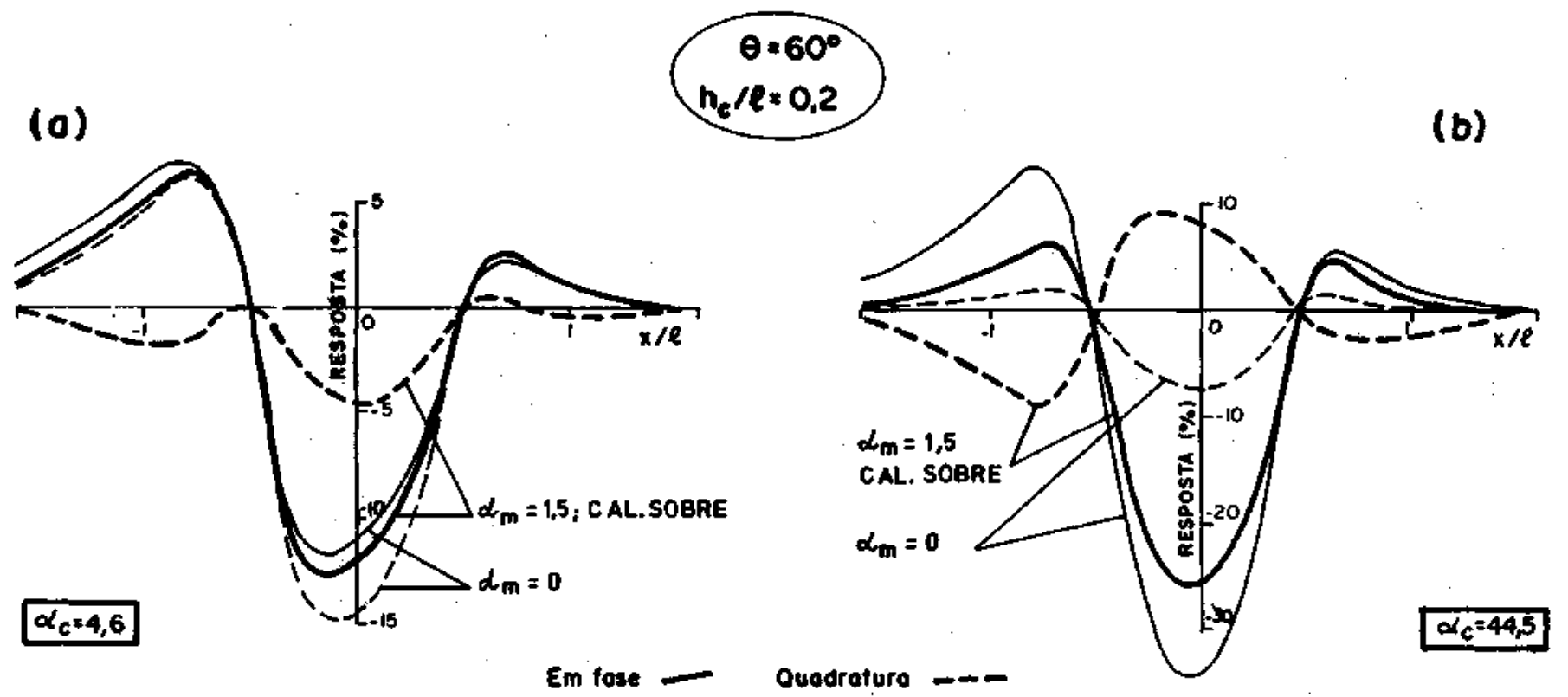

Figura 14 - Variação das respostas com o parâmetro de resposta do corpo $\left(\alpha_{c}\right)$. a. $\alpha_{c}=4,6$ e b. $\alpha_{c}=44,5$

Figure 14 - Variation of response profile with response parameter of the body $\left(\alpha_{c}\right)$. a. $\alpha_{c}=4,6$ and b. $\alpha_{c}=44.5$

refletindo a interação dos efeitos de pico extra e reversão da quadratura (Figs. 6 a 13).

Efeitos da cobertura x calibração das bobinas (CAL. SOBRE , CAL. FORA) Quando o sinal de referência é calibrado em zona onde afloram rochas resistivas (CAL. FORA), a simetrização da em-fase (Fig. 17a) e a reversão da quadratura (Fig. $1 \mathrm{~Tb}$ ) tomam-se mais efetivas do que quando a resposta é obtida com a calibração sobre o manto (CAL. SOBRE) e o deslocamento da base do perfil passa a existir (Figs. 17a e 17b). A calibração do sinal sobre a cobertura, por sua vez, favorece a formação do pico extra (Fig. 17b).

Nos AIDS é possível observar que as respostas obtidas com a calibração sobre a camada superficial, que compensa a redução de amplitude da anomalia introduzida por esta camada, bem como a rotação de fase, apresentam uma atenuação superior àquela observada para os perfis coletados com a calibração sobre aflorantes resistivas e um giro de fase inferior (Fig. 18); as respostas CAL. SOBRE podem, contudo, experimentar maior rotação de fase do que as respostas CAL. FORA, (caso $\alpha_{m}=0,5$, Silva 1981). A interpretação com um AID para condutor capeado por manto de parâmetro de resposta inferior ao real apontará, sempre, para um depósito mais profundo do que o real, mas a discrepância será maior se os perfis tiverem sido obtidos com a CAL. SOBRE do que com a CAL. FORA.

SÍNTESE DOS RESULTADOS A tabela 2 sintetiza resultados aqui mostrados bem como para coberturas superficiais de número de indução fraco a moderado (Lowrie \& West 1965 e Silva \& Verma 1986). A influência da cobertura superficial é dividida em efeitos de componente e de perfil associados, respectivamente, aos efeitos que são percebidos em uma única das componentes e aqueles que devem ser analisados considerando-se o trecho do perfil anômalo. Os primeiros podem auxiliar à interpretação qualitativa da anomalia. Os efeitos de perfil são especialmente úteis na interpretação quantitativa pelos diagramas de Argand, como da coleção Silva et al (1985).

Em termos práticos, a tabela 2 proporciona informações que podem ser expressas como segue.

Existindo a cobertura superficial:
1. Corpos de mergulho suave podem ser confundidos com corpos verticais a subverticais quando se analisa a componente em-fase (informação contida na linha "MERGULHO", coluna "SIMETRIZAÇÃO").

2. A probabilidade da resposta em-fase de um condutor inclinado ser confundida com a de um condutor vertical aumenta na freqüência alta. O mesmo ocorre quando se expande a distância entre as bobinas. Por outro lado, entre as anomalias em-fase obtidas com uma mesma freqüência e espaçamento segundo diferentes perfis que cruzam um condutor isolado inclinado, a de maior simetria pode ser a mais interessante para posterior verificação, porque pode indicar onde o condutor torna-se mais forte (informação da linha "NÚMERO DE INDUÇÃO", coluna "SIMETRIZAÇÃO").

3. Um aumento na simetria de uma anomalia de um perfil para outro pode significar uma menor profundidade para o condutor (linha "PROFUNDIDADE", coluna "SIMETRIZAÇÃO"). 4. A formação de pico extra indica que o condutor é inclinado ("MERGULHO", "PICO EXTRA").

5. O pico extra, que dificulta sobremaneira a quantificação da anomalia, pode ser eliminado, tanto pelo aumento como pela diminuição da freqüência e/ou espaçamento entre as bobinas ("PIÇO EXTRA", "NÚMERO DE INDUÇÃO").

6. Um condutor mais raso produz um pico extra mais acentuado ("PICO EXTRA", "PROFUNDIDADE").

7. A reversão da quadratura é maior quanto maior for a freqüência e/ou o espaçamento entre as bobinas. Por outro lado, entre as anomalias em quadratura obtidas com uma mesma freqüência e distância entre as bobinas ao longo de diferentes perfis que cruzam um condutor isolado inclinado, a anomalia de mais forte reversão pode ser a mais interessante para posterior verificação, porque pode' indicar onde o condutor torna-se mais forte ("NUMERO DE INDUÇÃO", "REVERSÃ̂").

Informações complementares:

I. O sentido do mergulho do corpo é o sentido da "cauda" da anomalia em-fase ou em-quadratura, bem como, considerando-se a anomalia em-fase, o sentido da área sob sua parte positiva que atinge o maior valor e do deslocamento do seu pico negativo (segundo parágrafo do item "Efeitos da cobertura x mergulho do corpo"); 

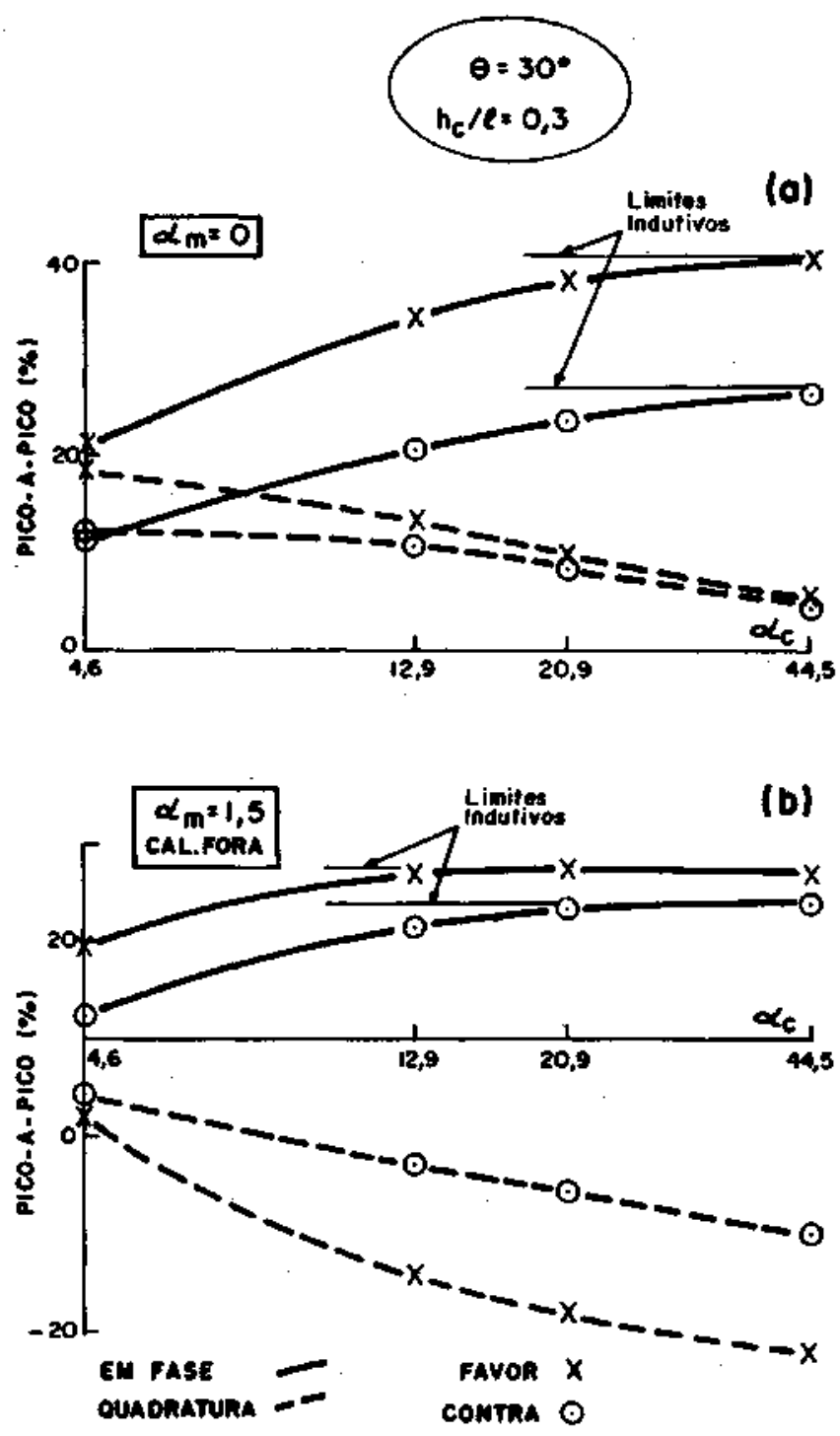

Figura 15 - Variação dos valores pico-a-pico com o parâmetro de resposta do corpo $\left(\alpha_{c}\right)$, a. sem a cobertura $\left(\alpha_{m}=\right.$ 0) e b. com a cobertura $\left(\alpha_{\mathrm{m}}=1,5\right)$

Figure 15 - Variation of peak-to-peak values with response parameter of the body (etc), a. when there is no overburden $\left(\alpha_{m}=0\right)$ and $\mathbf{b}$. when the overburden has a response parameter $\alpha_{m}=1,5$

II. A interpretação com um AID para condutor de mergulho superior ao real, capeado ou não pela cobertura superficial, aponta para um corpo mais raso e de maior condutância do que o verdadeiro, exceto quando, é utilizado um AJD-PPC obtido com o manto presente, quando a condutância interpretada será inferior a real (terceiro parágrafo do item "Efeitos da cobertura x mergulho do corpo").

As informações contidas na tabela, bem como as informações complementares, devem ser utilizadas de forma integrada para ter sentido prático. Combinando-se as informações fornecidas pelo efeito da cobertura sobre a emfase (itens 1, 2 e 3) pode-se, por exemplo, afirmar que as anomalias em-fase mais simétricas, obtidas sobre condutor inclinado, capeado pelo manto de intemperismo, com uma mesma freqüência e distância entre as bobinas, são as de maior interesse econômico, porque podem indicar onde o condutor torna-se mais forte e/ou raso. A combinação das informações 4,5 e 7 mostra que o pico extra indica que o condutor é inclinado, mas nem sempre que o condutor é

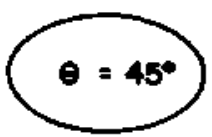

(a)
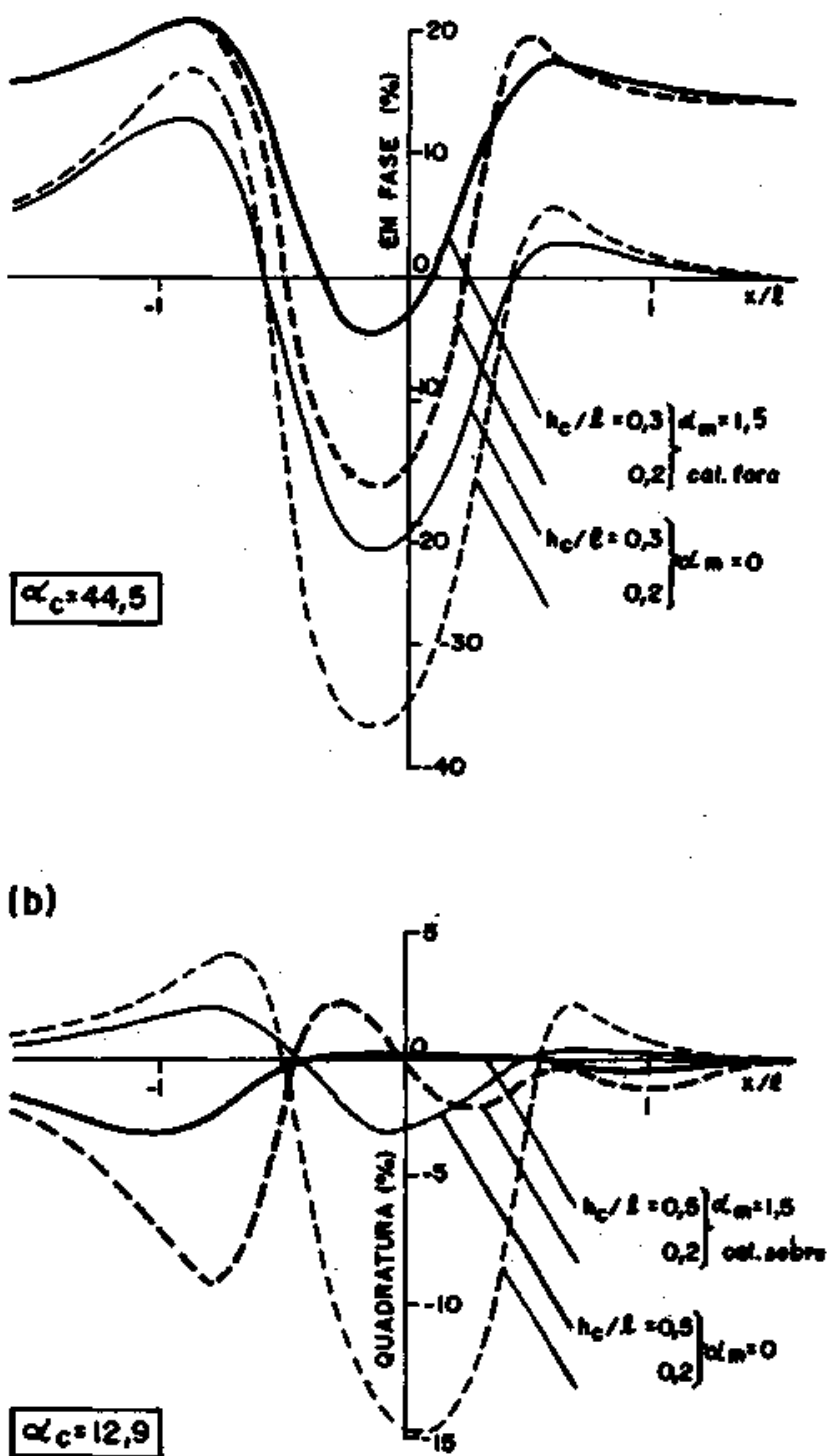

Figura 16 - Variação das respostas com a profundidade do corpo $\left(h_{c} / \ell\right)$. a. em-fase e b. quadratura

Figure 16 - Variation of response profile with depth of the body $\left(h_{c} / \ell\right)$. a. in-phase and $\mathbf{b}$. quadrature

inclinado aparece o pico extra; o aumento da freqüência ou do espaçamento entre as bobinas, por exemplo, pode eliminar completamente o pico extra, restando, apenas, o efeito de reversão. Com 5 e 7: entre os perfis obtidos com uma mesma freqüência e distância entre as bobinas, aquele em que o pico extra dá lugar a mais forte reversão pode indicar onde o condutor torna-se mais forte.

Os resultados apresentados, é conveniente frisar, são válidos para situações de campo passíveis de serem simuladas pelo arranjo experimental (vide item de mesmo nome e, para uma síntese das limitações experimentais, a Fig. 1).

DISCUSSÃO E CONCLUSÕES Resultados HLEM, obtidos pelo modelamento analógico e aqui apresentados, mostraram I. um efeito não-reportado, a simetrização da com- 


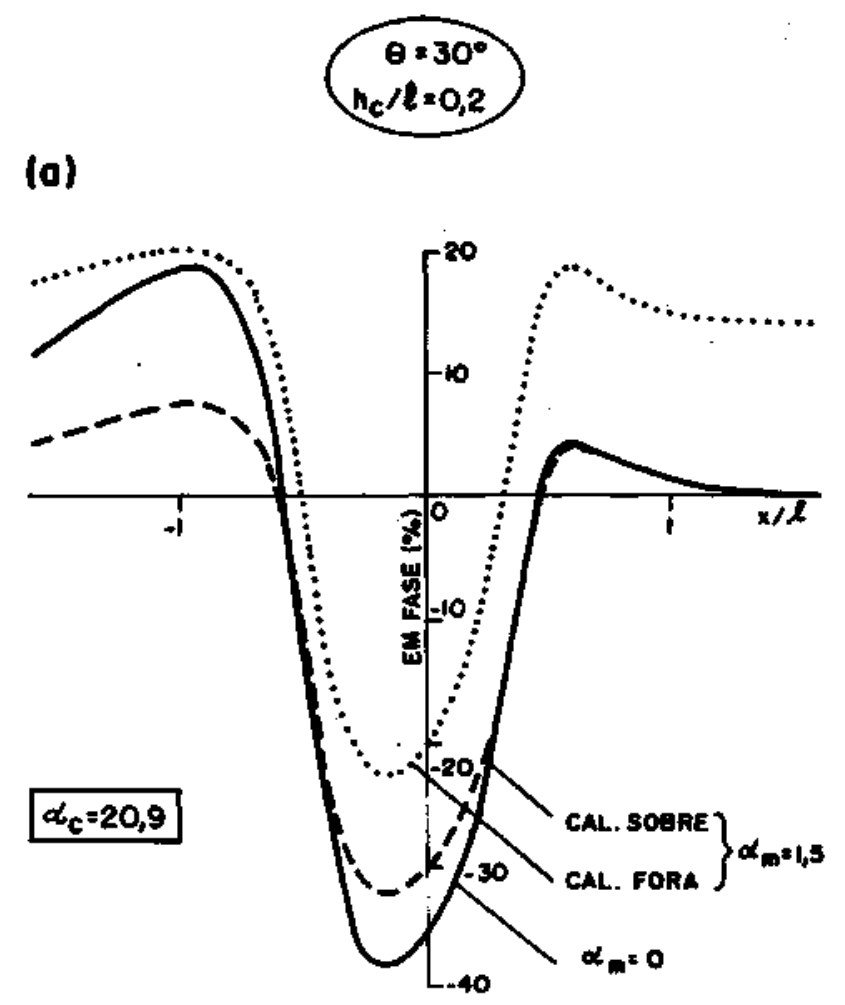

(b)

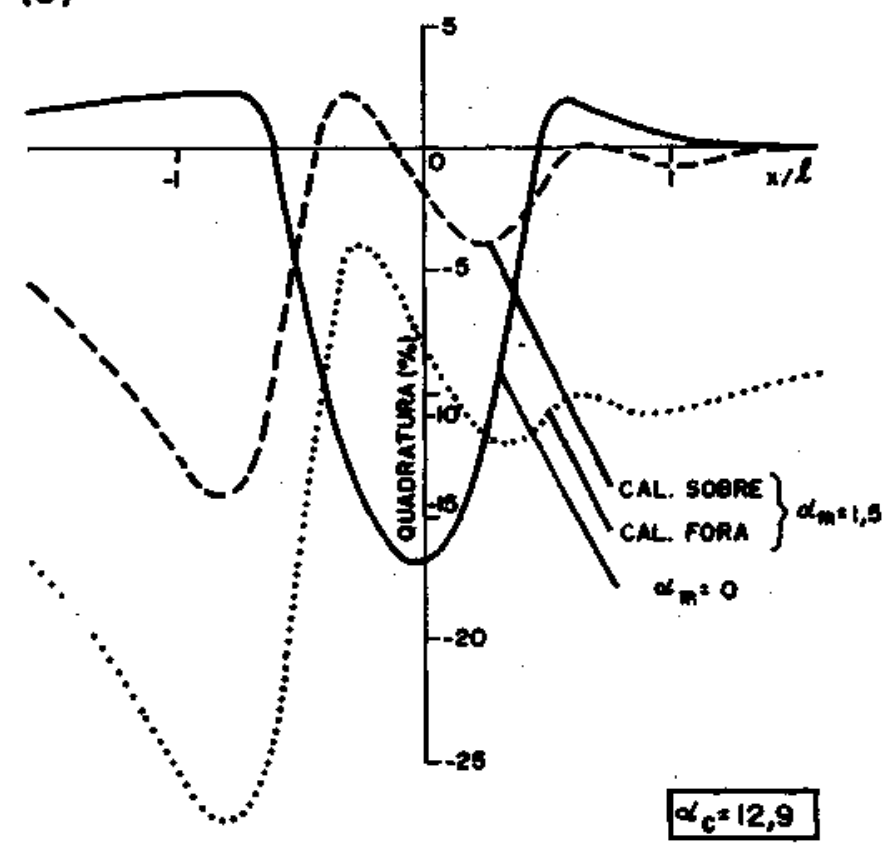

Figura 17 - Variação das respostas com a calibração do sistema (CAL SOBRE, CAL FORA), a. em-fase e b. quadratura Figure 17 - Variation of response profile with calibration of the loops (CAL. SOBRE: calibration with the overburden, CAL. FORA: calibration without the overburden), a. in-phase and $\mathbf{b}$. quadrature

ponente em-fase, provocado pela camada superficial sobre a resposta de condutor inclinado e II. relações entre os efeitos da camada superficial e parâmetros do problema. Primeiramente, abordam-se aspectos relevantes à compreensão da utilidade destas relações para a prospecção EM, bem como o aparente paradoxo que a existência de tais relações representa,

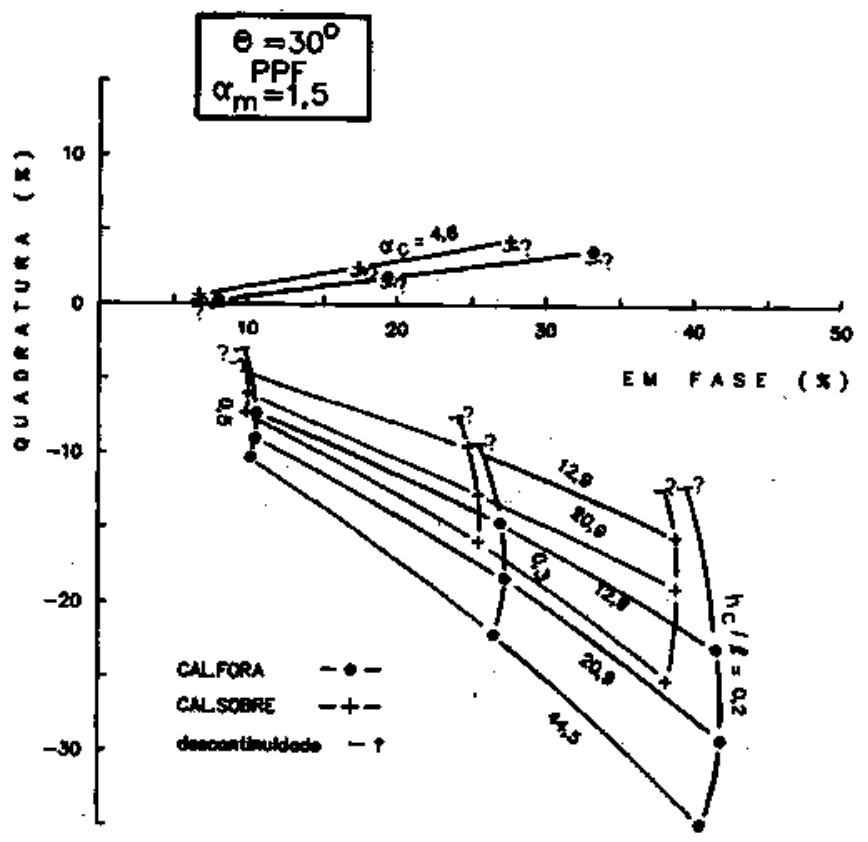

Figura 18 - Diagramas de valores pico-a-pico do lado a favor do mergulho do corpo para $\theta=50^{\circ}, \alpha_{\mathrm{m}}=1,5$ e calibração das bobinas fora e sobre o manto (CAL SOBRE e CAL FORA)

Figure 18 - Argand diagrams (peak-to-peak excursion of the components in the down dip side - PPF) for a body with dip angle $\theta=30^{\circ}$ when the overburden has a response parameter $\alpha_{m}=1,5$ and the loops are calibrated with and without the overburden (respectively, CAL. SOBRE and CAL. FORA)

porque significam a possibilidade de obter informações sobre o corpo de interesse a partir do ruído, uma vez que os efeitos do manto são considerados ruído do tipo geológico. Em

Tabela 2 - Efeitos da cobertura x parâmetros do modelo Table 2 - Overburden effects vs. model parameters

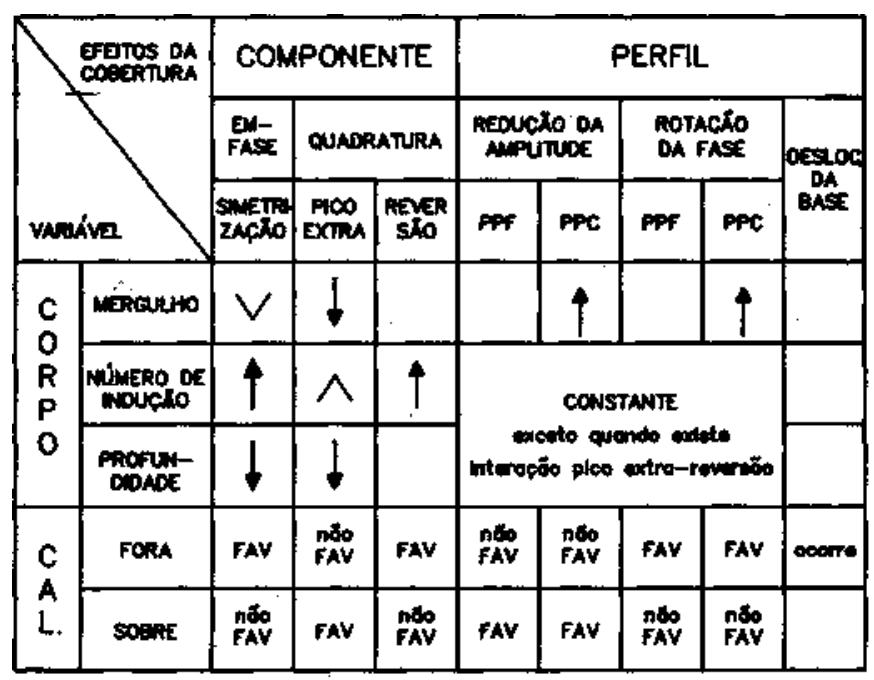

$\uparrow$ dirotamente proporclonol

$\downarrow$ inversomente proporcionol

A diretemente proporcional pora pequenos vilores inversomente, poro os demols

$\checkmark$ inversamente proporcional para pequerios valores e dretomente, poro os dempis

FAV fovorável 
seguida, a simetrização da em-fase e questões relacionadas são abordadas.

O objetivo do método EM é detectar e avaliar cada elemento da seção geoelétrica, logo separar o sinal devido ao alvo do ruído produzido pela cobertura e outras fontes de ruído (Hohmann \& Ward 1981 e Ward 1983). Na perseguição desse objetivo, que constitui um dos paradigmas da exploração EM (West \& Macnae 1984), surgiram os procedimentos de levantamento de dados com sistemas multifreqüências e multicanais de tempo de decaimento, com diferentes combinações de arranjos -de bobinas e espaçamentos.

O sinal de interesse, para poder ser separado do ruído geológico, deve estar associado a uma fonte de parâmetro de resposta superior ao parâmetro de resposta da fonte de ruído, mas uma superposição entre ambos não é rara (Ward 1983). A figura 19 reúne, sob a forma de histograma, 72 valores de condutância $(\sigma \mathrm{t})$ de diferentes depósitos de sulfetos extraídos de Bosschart (1961); Paterson (1961), Strangway (1966) e Questor Surveys Ltd. (s.d.). Cerca de $50 \%$ desses valores não são maiores do que $10 \mathrm{~S}$, um valor de condutância que a cobertura superficial pode atingir (Preston 1975). Conseqüentemente, as contribuições do corpo e do manto podem ser de distinção problemática.

McCracken (1983) aponta, para o alvo e à camada superficial da Austrália, os limites indutivos de, respectivamente, cerca de $100 \mathrm{~Hz}$ e 300-1000 Hz. A cobertura, portanto, para freqüências superiores a $300 \mathrm{~Hz}$, produz uma resposta dominante. Acima de $300 \mathrm{~Hz}$, cobertura e alvo exibem a mesma dependência da freqüência; a discriminação de ambos é, em conseqüência, impossível. Operações com freqüências de $1 / 10$ do limite indutivo da camada superficial (isto é,
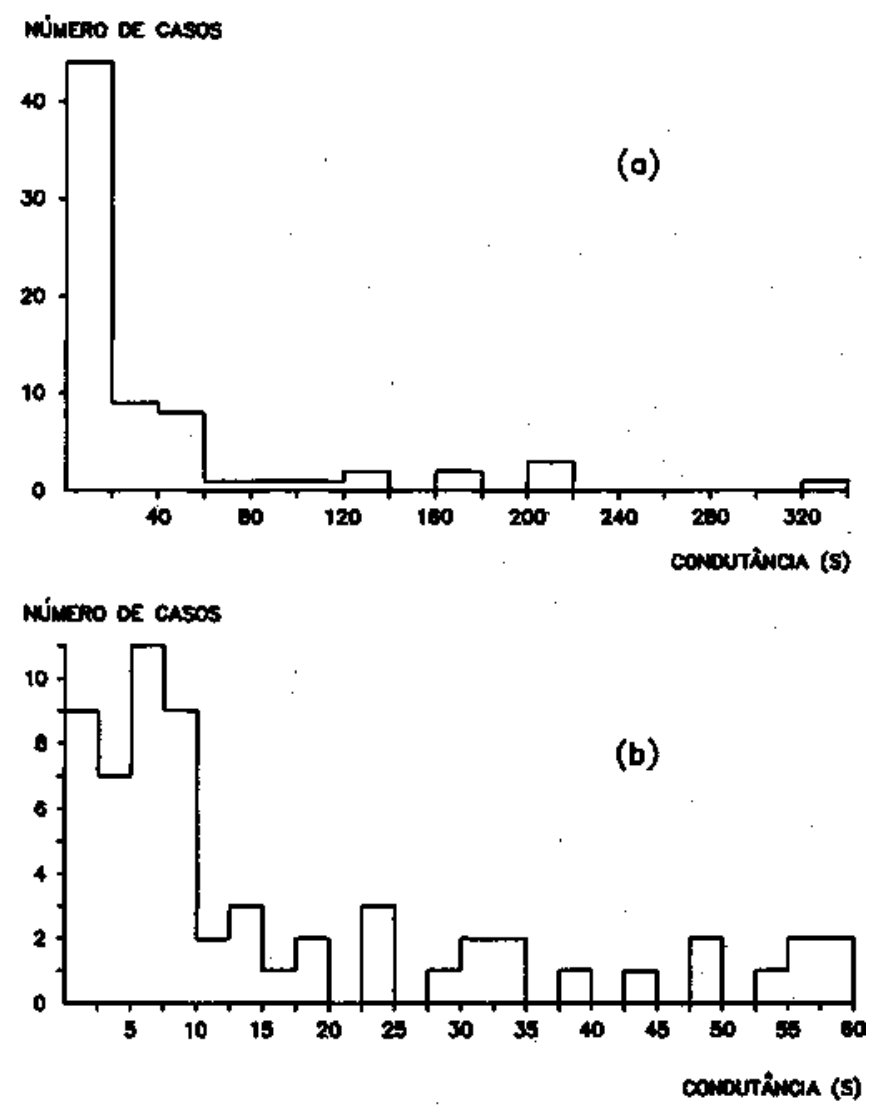

Figura 19 - Freqüencia das condutâncias de diversos depósitos de sulfetos. a. Distribuição total e $\boldsymbol{b}$. distribuição no intervalo de 0 a $60 \mathrm{~S}$

Figure 19 - Conductances of some sulfide deposits, a. Total distribution and b. distribution between $0-60 \mathrm{~S}$
$30 \mathrm{~Hz}$ para o modelo australiano) reduzem enormemente, por um fator de 10 , a resposta dessa camada, enquanto a resposta do alvo é reduzida por um fator de 2 . A grande maioria dos equipamentos do domínio da freqüência, contudo, operam com todas as suas freqüências ou a maioria delas, acima de $300 \mathrm{~Hz}$.

Em adicional, atualmente, o método EM reconhece interações, não meras superposições, de correntes induzidas em cada um dos elementos do problema de exploração EM (Hohmann \& Ward 1981, Ward 1983): as correntes induzidas no corpo induzem correntes no ambiente e vice-versa, com as conseqüentes modificações do campo; as correntes que circulam no ambiente podem modificar suas trajetórias, por causa da presença do condutor principal, cerrando seus circuitos através deste; e as correntes que circulam no condutor, de modo análogo, podem completar suas trajetórias através do ambiente que as rodeia (Orellana 1974). A primeira interação, puramente indutiva, está sempre presente. A interação que representa uma mudança na geometria da trajetória da corrente, depende da existência de descontinuidade na condutividade entre o ambiente e o alvo (caso galvânico); mudanças na trajetória da corrente podem, contudo, ser produzidas por uma interação puramente indutiva (Sarma \& Maru 1971). A figura 20 ilustra, de forma esquemática, as interações para o modelo corpo, manto e corpo + manto.

As interações corpo-manto desviam as medidas da resposta devida unicamente ao corpo, sendo consideradas parte do ruído $\left(\mathrm{S}_{\mathrm{cm}}^{\mathrm{I}}\right.$ e $\mathrm{S}_{\mathrm{cm}}^{\mathrm{II}}$, Fig. 20). Por outro lado, como o corpo participa do processo, variações em seus parâmetros afetam tais interações. Informações sobre o corpo encontram-se, conseqüentemente, dispersas no chamado ruído, e podem ser capturadas, como mostrado por Silva (1989) e, em detalhe, neste trabalho, ao examinar-se as relações entre os efeitos da camada superficial e os parâmetros do problema. A análise do ruído EM significa maximizar a obtenção de informações contidas na resposta que é tratada como um todo (a separação da resposta não se aplica por razões já expostas) podendo representar, portanto, um caminho adicional para a compreensão das anomalias EM e, dessa forma, para a distinção dos alvos de interesse econômico.

A simetrização da em-fase, por sua vez, corresponde à saturação da componente em-fase, segundo limites de indução convergentes, tomando-se os valores pico-a-pico de ambos os lados da anomalia (Fig. 15); variações no mergulho do condutor principal vão, em conseqüência, afetar a resposta HLEM em-fase de forma não tão acentuada como seria o esperado. Efeito análogo foi descrito por Verma (1972), Gaur et al (1972), Gaur \& Verma (1973) e Verma \& Gaur (1975) para situações de prospecção em que o alvo encontra-se imerso em rocha encaixante com alguma condutividade e associado à concentração, pelo corpo principal, da corrente induzida na encaixante condutiva, com o conseqüente aumento na densidade de corrente através do condutor, uma extensa feição absorve, ademais, uma quantidade maior de energia EM do que o corpo considerado isoladamente, favorecendo o incremento da densidade de corrente. A simetrização da em-fase pela encaixante foi associada ao fenômeno de aumento da resposta do alvo em ambiente condutivo, proposto como "blindagem negativa" por Negi (1967), a partir do estudo dos efeitos do halo de sulfetos disseminados que pode se desenvolver ao redor do corpo de sulfetos maciços. O fenômeno tem despertado importantes polêmicas quanto ao seu significado físico (Roy 1970, Sarma \& Maru 1971 e Kamenetiskiy \& Timofeev 1978), ao mesmo tempo que tem sido verificado em estudos sobre os efeitos da encaixante (Sarma \& Maru 1971, Verma 1972, Gaur \& Verma 1973, Verma \& Gaur 1975) e do manto deintemperismo(Joshi et al. 1984).

No fenômeno de concentração de corrente (current gathering), as correntes induzidas no ambiente condutivo (encai- 


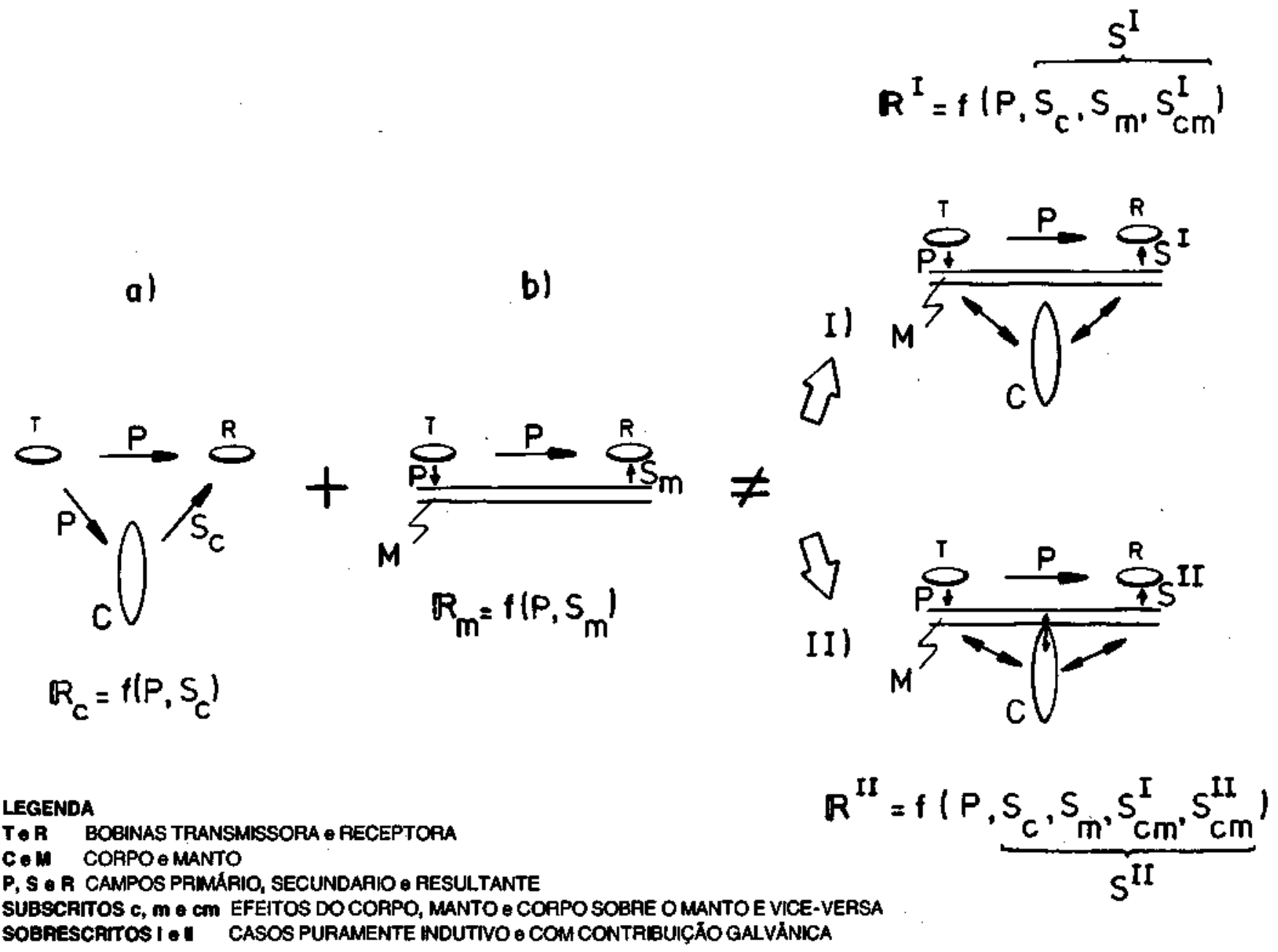

Figura 20 - Comparação esquemática entre campos EM. O campo total resultante (R) para o modelo corpo-manto não é obtido por meio da superposição de a. campo devido ao corpo (Rc) com o b. campo devido ao manto ( $\left.R_{M}\right)$. No caso I, é necessário considerar que as correntes induzidas no corpo induzem correntes na cobertura e vice-versa $\left(\mathrm{S}_{\mathrm{cm}}^{\mathrm{I}}\right)$. No caso II, corpo em contato galvânico com a cobertura, ocorre, ademais, que as correntes induzidas fluem por entre ambos ( $\left.\mathrm{S}_{\mathrm{cm}}{ }_{\mathrm{l}}\right)$

Figure 20 - Schematic comparison between EM fields. The resultant field (R) due to the model body-overburden is not the superposition of the field due to the a. body $(\mathrm{Rc})$ and b. overburden $\left(\mathrm{R}_{\mathrm{M}}\right)$. In the case $\mathrm{I}$, it is necessary to consider that the currents induced in the body induce currents in the overburden and viceversa $\left(S_{c M}^{I}\right)$. In the case II, the body and the overburden are in galvanic contact, therefore the induced currents flow from one model to the other $\left(S^{I I}{ }_{c M}\right)$

xante, halo ou manto) podem fluir para o corpo de maior condutividade, porque as entidades geológicas estão em contato galvânico (caso II, Fig. 20). A simetrização da em-fase apresentada neste trabalho, contudo, foi obtida com o condutor principal isolado da cobertura superficial.

A explicação para o efeito de simetrização deve levar em consideração os seguintes aspectos:

- a concentração das linhas de corrente dentro do corpo principal deve se dar muito próxima do topo do corpo, segundo uma bobina vertical (Verma 1972, Gaur \& Verma 1973, Verma \& Gaur 1975) e

- os limites indutivos para o modelo corpo-manto são de valor inferior aos limites indutivos obtidos sem o manto (Fig. 15); a atenuação da energia provocada pela presença do manto projeta-se essencialmente na em-fase, que perde em intensidade e, portanto, em penetração - o que corrobora com a aproximação do condutor por uma bobina próxima de seu topo.

A origem da simetrização, no caso estudado, deve ser associada à blindagem ("positiva") do manto $\left(\mathrm{S}_{\mathrm{M}}\right.$, Fig. 20$)$ e à interação entre correntes induzidas no condutor e na cobertura
$\left(\mathrm{S}_{\mathrm{CM}}^{\mathrm{I}}\right.$, Fig. 20) com as subseqüentes re-orientações do vetor campo no espaço. Mudanças na geometria da trajetória da corrente, como já foi mencionado, podem ser provocadas, tanto pela descontinuidade da condutividade entre o alvo e o manto como por interação puramente indutiva.

Finalmente, é interessante analisar o motivo da quadratura obtida na presença do manto não apresentar nenhum efeito semelhante à simetrização da em-fase, que corresponde a uma perda de sensibilidade da componente.

O campo magnético sofre, ao atravessar uma profundidade igual ao skindepth $\delta(=500 \sqrt{1 / f \sigma})$, uma rotação de $180^{\circ} \mathrm{e}$ uma atenuação em seu valor original de 1/e (37\%). Com a folha de aço utilizada na simulação da cobertura $(\sigma=1,387 \mathrm{x}$ $10^{6} \mathrm{~S}$ e $\mathrm{t}=0,812 \times 10^{-3} \mathrm{~m}$, Tab. 1$)$, por exemplo, o campo magnético sofre, ao atravessá-la, uma rotação de $9^{\circ}$ e uma atenuação de $2 \%$; o mesmo ocorre com o campo que alcança a bobina receptora após passar pelo manto. O campo, ao atravessar a camada superficial, sofre atenuação, mas é, principalmente, deslocado na fase. Esta variação na fase é, no entanto, reduzida; o campo secundário que chega à bobina 
receptora, além do atraso $\pi / 2$, sofre, portanto, um giro pequeno, de modo que ele é, essencialmente, em-quadratura com o campo primário.

A folha de aço $\left(\sigma=1,418 \times 10^{6} \mathrm{~S}\right.$ e $\mathrm{t}=2,570 \times 10^{-3} \mathrm{~m}$, Tab. 1) utilizada na simulação do corpo de menor condutância gira o campo de $29^{\circ}$ e atenua-o em $6 \%$. No caso do condutor mais forte (placa de Al de $\sigma=33,126 \times 10^{6} \mathrm{~S}$ e $\mathrm{t}=1,008 \times 10^{-3} \mathrm{~m}$, Tab. 1), a rotação é de $54^{\circ}$ e a atenuação de $11 \%$.

A figura 21 mostra estes valores lançados em diagrama vetorial. Enquanto as correntes no manto estão predominantemente em-quadratura com o campo primário, as correntes no corpo podem estar em-quadratura ou em-fase. Embora o campo total resultante (ou secundário) não corresponda à superposição do campo resultante (ou secundário) devido ao condutor principal com o campo resultante (ou secundário) gerado pela camada superficial, a presença desta camada vai significar, pelo menos, a introdução na resposta de efeitos em-quadratura. Tem sido apontado que, em terrenos acidentados, a componente em-fase, mais afetada pela topografia, pode ser ignorada com o risco, contudo, de se perder corpos fortemente condutivos que não produzem anomalias em-quadratura significativas (Keller \& Frischknecht 1979). Com a cobertura condutiva, o risco inexiste: a quadratura manifesta-se para condutores fortes, enquanto a sensibilidade da em-fase é reduzida.

Agradecimentos Este trabalho tornou-se possível graças ao apoio da FINEP e do CNPq na montagem e manutenção da infra-estrutura laboratorial utilizada, e ao incentivo, sob a forma de bolsa de pesquisa, aos primeiro e segundo autores, do CNPq e da FADESP, respectivamente. A essas entidades, bem como ao prof. dr. Qm Prakash Verma, pelas valiosas observações oferecidas na fase inicial da interpretação dos resultados, ao geofísico João Carlos Ribeiro Cruz, pelo auxílio

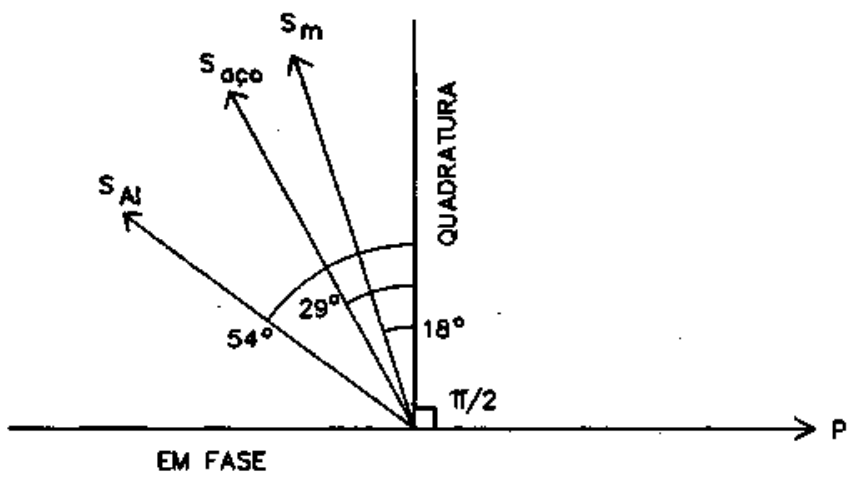

$P$

$\mathrm{S}_{\mathrm{m}} \quad$ CAMPO SECUNDÁRIO DEVIDO AO MANTO

$S_{\text {aço }}$ e $S_{A I} \quad$ CAMPOS SECUNDÁRIOS DEVIDOS AOS CORPOS DE MENOR E MAIOR CONDUTÂNCIA

Figura 21 - Relação entre o campo primário após atravessar o manto e corpos de menor e maior condutância utilizados no experimento em diagrama vetorial

Figure 21 - Relation between the secundary field due to the overburden $\left(\mathrm{S}_{\mathrm{m}}\right)$ and bodies of the lowest and highest conductance used in the experiment $\left(\mathrm{S}_{\mathrm{aco}}\right.$ and $\mathrm{S}_{\mathrm{AL}}$, respectively)

no levantamento dos perfis teóricos, e ao especialista em eletrônica Ralph E. Strobl, pelas sugestões e ajuda na compilação e ilustração dos resultados, os autores dirigem seus agradecimentos.

\section{REFERÊNCIAS BIBLIOGRÁFICAS}

BOSSCHART, R.A. 1966. On the ocurrence of resistivity geological conductors. Geoph. Prosp., 9(2):203-212.

GAUR, V.K. \& VERMA, O.R 1973. Enhancement of electromagnetic anomalies by a conducting overburden II. Geoph Prosp., 21(1): 159-184.

GAUR, V.K. VERMA, O.P. GUPTA, C.P. 1972. Enhancement of electromagnetic anomalies by a conducting overburden. Geoph. Prosp., 20(3):580-604.

GRANT, F.S. \& WEST, G.F. 1965. Interpretation theory in Applied Geophysics. New York, McGraw-Hill. $584 \mathrm{p}$.

HOHMANN, G.W. \& WARIX S.H. 1981. Electrical methods in Mining Geophysics. EC. Geol, 75 $^{\text {th }}$ Anniversary: 806-828.

ISSLER, R.S. \& SILVA, G.G. 1980. The Seis Lagos carbonatite complex. In: CONOR. BRAS. GEOL., 31. Camboriu, 1980. Anais... Camboriú, SBG. v.3, p. 1564-1573

JOSHI, M.S.; GUPTA, O.R; NEGI, J.G. 1984. Scale model response of a thin vertical conductor below a conductive, inductive, or laterally inhomogeneous overburden layer. Geophysics, 49(12):2159-2165.

KAMENETISKIY, F. M. \& TIMOFEEV, V.M. 1978. Negative electromagnetic screening. Izv., Earth Phys., 14(3):226-230.

KELLER, G.V. \& FRISCHKNECHT, F.C. 1979. Electrical methods in geophysical prospecting. Oxford, Pergamon. $523 \mathrm{p}$.

LAJOIE, J.J. \& WEST, G.F. 1977. Short note: two selected field examples of EM anomalies in a conductive environment. Geophysics, 42(3):655-660.

LOWRIE, W. \& WEST, G.F. 1965. The effect of a conducting overburden on electromagnetic prospecting measurements. Geophysics, 30(4):624632.

McCRACKEN, K.G. 1983. Electrical Geophysics in the arid and tropical zones of Australia. In: INT. SYMP. APLLIED GEOPH. TROP. REGIONS, 1. Belém, 1982. Proceedings... Belém, SBGf. p. 87-109.

NAIR, M.R.; BISWAS, S.K.; MAZUMDAR, K. 1968. Experimental studies on the electromagnetic response of tilted conducting half-planes to a horizontal-loop prospecting system. Geoexpl., 6(4):207-244.

NEGI, J.G. 1967. Electromagnetic screening due to a disseminated spherical zone over a conducting sphere. Geophysics, 32(1):69-87.

ORELLANA, E. 1974. Prospección geoeléctrica por campos variables. Madrid, Paraninfo. $571 \mathrm{p}$.

PALACKY, G.J. 1975. Interpretation of INPUT AEM measurements in areas of conductive overburden. Geophysics, 40(3):490-502.
PALACKY, G.J \& KADEKARU, K. 1978. Efeito do intemperismo tropical nos levantamentos eletromagnéticos. In: CONGR. BRAS. GEOL., 30. Recife, 1978. Anais... Recife, SBG. v.5, p. 2323-2330.

PALACKY, G.J. \& KADEKARU, K. 1979. Effect of tropical weathering on electrical and electromagnetic measurements. Geophysics, 44(1):69-88.

PALACKY, G.J. \& SENA, F.O. 1979. Conductor identification in tropical terrains - case histories from the Itapecuru Greenstone belt, Bahia, Brazil. Geophysics, 44(12): 1941-1962.

PARASNIS, D.S. 1971. Analysis of some multi-frequency, multi-separation electromagnetic surveys. Geoph. Prosp., 19(2):163-I79.

PATERSON, N.R. 1961. Experimental and field data for the dual-frequency phase-shift method of airborne electromagnetic prospecting. Geophysics, 26(5):601-617.

PATERSON, N.R. 1967. Exploration for massive sulphides in the Canadian Shield. Econ. Geol. Report. 26: 275-289.

PRESTON, B. 1975. Review - Difficulties for the electromagnetic method in Australia. Geoexpl, 13(1):29-43.

QUESTOR SURVEYS Ltd. s.d. The Questor Method. Mississauga, Ontario Case histories: Brouillan discovery, Detour River discovery, Lessard deposit, Magusi River discovery, New Insco discovery, Rail zone, Reed Lake discovery, Thierry deposit, Yava zone. Canadá, Questor Surveys Ltd. (Folh. propaganda).

ROY, A. 1970. On the effect of overburden on electromagnetic anomalies a review. Geophysics, 35(4):646-659.

S ARMA, D.G. \& MARU, V.M. 1971. A study of some effects of a conducting host rock with a new modelling apparatus. Geophysics, 36(1):166-183.

SILVA, L.M.C. 1981. Efeitos do manto de intemperismo sobre anomalias eletromagnéticas provocadas por corpos tabulares inclinados - um estudo através de modelamento reduzido. Belém. 115 p. (Dissertação de mestrado, UFPA/NCGG).

SILVA, L.M.C. 1989. Relations between EM effects of the overburden and unknown parameters of the model. In: EUROPEAN ASSOC. EXPL. GEOPH. MEETING AND TECHNICAL EXHIBITION, 51. Berlin, 1989. Technical Programme and Abstracts of Papers... Berlin, EAEG. p. 215-216.

SILVA, L.M.C. \& VERMA, O.R 1986. Efeitos do manto de intemperismo sobre anomalias eletromagnéticas (HLEM) geradas por corpos tabulares inclinados. Rev. Bras. Geoc., 16(3):285-293. 
SILVA, L.M.C.; VERMA, O.P.; GALVÀO, M.V.O. 1985. Curvas de interPretação eletromagnética (HLEM) para corpos tabulares sob manto de intemperismo. Belém, UFPA/GEU-CG/Dpt ${ }^{\circ}$ Geof. 91p.

STRANOWAY, D.W. 1966. Electromagnetic parameters of some sulfide orebodies. In: HANSEN, D.A. et al. Eds. Mining Geophysics. USA, SEO. v. 1, p. 227-242

TELFORD, W.M; GELDART, L.P.; SHERIFF, R.E.; KEYS, D.A. 1981. Applied Geophysics. Cambridge, Cambridge University. $860 \mathrm{p}$

VERMA, O.P. 1972. Electromagnetic model experiments simulating conditions encountered in geophysical prospecting, Índia. 143 p. (Tese de doutorado, Umvenky of Roorkee).

VERMA, O.P. \& OAUR, V.K. 1975. TramfonnMion of electromagnetic anomalies brought about by a conducting host rock. Geophysics, 40(3):473-489.
WARD, S.H. 1983. State of the art perspective for Mining Geophsics. In: WT, SYMP. APPLIED GEOPH.TROP. REGIONS, 1.Belém, 1982. Proceedings... Belém, SBGf. p. 3-86.

WEST, G.F. \& MACNAE, J.C. 1984, Electromagnetics for geophysical exploration. Toronto, Noto. $101 \mathrm{p}$.
MANUSCRITO A615

Recebido em 15 de agosto de 1989

Revisão do autor em 08 de maio de 1990 Revisão aceita em 15 de maio de 1990 